\title{
Thermodynamics of shape-memory alloys under electric current
}

\author{
Tomáš Roubíček and Giuseppe Tomassetti
}

\begin{abstract}
Phase transformation in shape-memory alloys is known to cause electric resistivity variation that, under electric current, may conversely influence Joule heat production and thus eventually the martensitic transformation itself. A thermodynamically consistent general continuum-mechanical model at large strains is presented. In special cases, a proof of the existence of a weak solution is outlined, using a semidiscretization in time.
\end{abstract}

Mathematics Subject Classification (2000). 35K55; 74A15; 74N10; 80A17.

Keywords. Generalized standard materials, heat equation, Joule heat, electrically conductive media, martensitic transformation, weak solution.

\section{Introduction}

Shape-memory alloys (=SMAs) as so-called active (or smart) materials have been subjected to intensive theoretical and experimental research during the past decades. They are typically intermetallic compounds whose atomic lattice is based on chemical bonds and exhibit specific hysteretic stress/strain/temperature response, which is called shape-memory effect. This is related with the phenomenon that atoms tend to be arranged in different crystallographic configurations (in particular, having different symmetry groups) depending on temperature. Higher temperatures leads to a higher-symmetry (typically cubic) lattice referred to as the austenite phase while lower temperature leads to a lower-symmetric grid (typically tetragonal, orthorhombic, monoclinic, or triclinic) called the martensite phase which may occur in several variants, namely $3,6,12$, or 4 in the mentioned cases, respectively. Specific case is a rhomboedric phase (a so-called R-phase), having 4 variants with distortion continuously depending on temperature.

SMAs are electrically conductive and it is very specific that their electric resistivity varies in dependence not only on temperature but mainly on the symmetry of the lattice. This variation may be quite markable, cf. e.g. [19, 33] where variation of tens of percents within transformation austenite to martensite or to R-phase is experimentally documented on NiTi. This may create specific effects of two sorts:

o different electric resistivity may easily be exploited to detect phase transformation; to this goal, only a very small electric current can be used without causing any coupling effects,

$\circ$ actuating phase transformation in SMA wires is often executed by heating by electric current; then coupling effects caused by phase-dependence of electric resistivity occur.

This research has been facilitated by the European project MRTN-CT-2004-505226 "Multi-scale modelling and characterisation for phase transformations in advanced materials". The work was also supported by "Nečas center for mathematical modeling" LC 06052 (MŠMT ČR), and the Project "Modellazione fisico-matematica dei continui elettro-attivi" (INdAM-GNFM, Italy), and partly supported also by the grants A 1075402 (GA AV ČR), and MSM 21620839 and 1M06031 (MŠMT ČR), 201/06/0352 (GA ČR), and from the research plan AV0Z20760514 (С̆R). 
So far, rather indirect experiments documenting phase-dependence of electric resistivity on strain or temperature in specifically loaded wires rather than on lattice state itself are at disposal, see e.g. [14, 21, 33, 54, 55]. Moreover, attempts for modelling this effect are so far rather speculative, based on counting serial arrangement of resistors [33, Sect.3.2] although a parallel arrangement is, in principle, quite equally justified especially in bulks.

One of the goal of this article is to develop a general rational thermodynamically consistent continuum mechanical model in Section 2. To this aim, we combine several generally accepted concepts, namely hyperelastic materials at large strains and the so-called generalized standard solids (due to Halphen and Nguyen [20]) with internal parameters involving here a vectorial order parameters (of the type as in Fremond's model [16]) like in phase-field models. We also adopt concept of dissipative forces having (pseudo)potentials, some of them may be degree-1 homogeneous to describe phenomenologically activated, rate independent effects which are typical within phase transformation. Eventually, we consider coupling with electric current through Onsager-type [34] cross-effects and production of heat through Joule effects. In particular, the general model thus counts with phase dependence not only of electric resistivity (or, equally, conductivity) but also heat conductivity. Beside applying these concepts, we present the model in a full thermodynamical context. This general concept, presented in Section 2, can be applied (possibly with some minor modifications) to various situations as electrically-conductive magnetostrictive materials or material exhibiting giant magnetic resonance.

Anyhow, as outlined above, in Section 3, we focus on materials exhibiting solid-solid phase transformations with heat and electricity conduction and we select a special model that hits the most important phenomena mentioned above and still allows for a rigorous mathematical analysis, outlined then in Section 4. In particular, we have to cope with multi-well stored energies (like in Falk's model [13]), which is related with a possible co-existence of several phases or phase variants; for this concept and examples in specific cases we only refer to e.g. $[5,6,23,24,26,40,46]$ but we do not have ambitions to mention it in details here. We will take into account so-called "capillarity-like" 2nd-order effects, as well as the "viscosity-like" effects that usually accompany them. Models with capillarity have been studied in $[22,36,37,38]$. For completeness, let us mention that models with no capillarity were studied in $[10,32,56]$, while models with no viscosity have been studied in $[1,18,51,52]$, cf. also [9, Chap.5].

In principle, the rationally based model may allow for identification of phasedependence of electric resistivity from experimental data. Also, the analysis presented in Section 4, based on a semi-discretization scheme, may lead after further discretization and effort to an implementable numerical strategy, cf. Remark 4.3 below.

\section{A general model for electrically conductive generalized standard materials}

In this section we outline a continuum theory that describes the thermodynamics of electrically conductive deformable continua with internal parameters. In addition to the standard mechanical forces, which expend power over motions of the material points of the body, we include a system of microforces and microstresses, which expend power over time variations of the order parameters. Moreover, in order to account for capillarity effects, that is, energy-storage that depends on the second derivative of the displacement, we introduce a mechanical hyperstress. Each system of forces has its own balance statement, which can be derived from the principle of virtual powers. To take temperature into account, we also include a balance of 
internal energy, and we postulate an entropy imbalance which we use, through a standard procedure due to Coleman and Noll [11], to rule out thermodynamically inconsistent constitutive choices. By combining the balance equations with the constitutive equations we obtain a quite general system of partial differential equations or inclusions, which describes generalized standard materials with heat and electricity conduction. This framework may, in principle, be used to describe not only SMAs, but also other materials with internal parameters, such as for example magnetostrictive materials.

Let $\Omega \subset \mathbb{R}^{3}$ be a bounded domain, assumed smooth for the purpose of the derivation of the model, although later in (4.2a) more general domains are admitted. In fact, when the domain has sharp edges, hyperstresses may involve concentrated forces [41]. Dealing with these issues is beyond the scope of this paper, however. We identify $\Omega$ with the reference shape of the body, typically a stress-free basic configuration; in our prominent application in Sect. 3, the adjective "basic" will refer to austenite. We consider a fixed time horizon $T>0$, we set $I=(0, T)$, and we denote by $y: I \times \Omega \rightarrow \mathbb{R}^{3}$ the time-dependent deformation of the body. We write $F=\nabla y$ and we denote by $u(t, x)=y(x)-x$ the displacement of $x \in \Omega$ at time $t \in I$. The deformation gradient is given by $F=\nabla y=\mathbb{I}+\nabla u$, where $\mathbb{I} \in \mathbb{R}^{3 \times 3}$ denotes the identity matrix. We also write $e(u)=\frac{1}{2}\left(\nabla u^{\top}+\nabla u\right)=: \operatorname{sym}(\nabla u)$. To keep trace of phase transformation, we introduce a vectorial order parameter $\lambda: I \times \Omega \rightarrow \mathbb{R}^{L}$, where $L$ is a positive integer. In addition to the standard stress $\sigma: I \times \Omega \rightarrow \mathbb{R}^{3 \times 3}$, we introduce the hyperstress $H: I \times \Omega \rightarrow \mathbb{R}^{3 \times 3 \times 3}$ that accounts for "capillarity-like" effects, and for the corresponding dissipation mechanisms. The force balance in the reference configuration is:

$$
\varrho \ddot{y}-\operatorname{div}(\sigma-\operatorname{div} H)=f,
$$

where $\varrho>0$ is the referential mass density and $f$ is the distance force per referential unit volume; here and henceforth, as a rule, each superimposed dot denotes the partial derivative with respect to time, hence in particular $\ddot{y}=\frac{\partial^{2}}{\partial t^{2}} y$. In the spirit of $[16,17]$, we regard the evolution of $\lambda$ as being driven by a system of internal microforces. We require these microforces to satisfy the balance law

$$
\operatorname{div} M-m=\mathbf{0} \text { on } \partial \Omega,
$$

where $M: I \times \Omega \rightarrow \mathbb{R}^{L \times 3}, m: I \times \Omega \rightarrow \mathbb{R}^{L}$ are, respectively, the microstress and the internal microforce. The balance of internal energy takes the form:

$$
\dot{\varepsilon}+\operatorname{div} q+j \cdot \nabla \phi-\sigma: \dot{F}-H: \nabla \dot{F}-m \cdot \dot{\lambda}-M: \nabla \dot{\lambda}=0,
$$

where " : " denotes the scalar product of tensors of the 3rd or higher order, while ":" or "." is used more conventionally for the 2nd-order tensors or vectors, respectively. Here $\varepsilon$ is the (specific) internal energy, $q$ and $j$ are, respectively, the material heat flux and the electric current flux, and $\phi$ is the material scalar potential [53]. The Kirchhoff law takes the form:

$$
\operatorname{div} j=0 .
$$

The local form of the Clausius-Duhem inequality is:

$$
\theta \dot{\eta} \geq q \cdot \nabla \theta-\operatorname{div} q
$$

We introduce the free energy:

$$
\psi:=\varepsilon-\eta \theta,
$$

and we combine the Clausius-Duhem inequality with the balance of energy to obtain:

$$
\dot{\psi}+\eta \dot{\theta} \leq-\theta^{-1} q \cdot \nabla \theta-j \cdot \nabla \phi+\sigma: \dot{F}+H: \nabla \dot{F}+m \cdot \dot{\lambda}+M: \nabla \dot{\lambda} .
$$


The fields to be constitutively specified are $\psi, \eta, q, \sigma, H, m, M$, and $j$. We assume that each of these fields, let us denote it generically by $\mathscr{F}$, depends on the state variables

$$
\Lambda^{\mathrm{eq}}:=(\theta, F, \nabla F, \lambda, \nabla \lambda), \quad \Lambda^{\text {neq }}:=(\nabla \theta, \nabla \phi, \dot{F}, \nabla \dot{F}, \dot{\lambda}, \nabla \dot{\lambda})
$$

through a response function $\widetilde{\mathscr{F}}\left(\Lambda^{\mathrm{eq}}, \Lambda^{\text {neq }}\right)$. We also define $\widetilde{\mathscr{F}}^{\mathrm{eq}}\left(\Lambda^{\mathrm{eq}}\right)=\widetilde{\mathscr{F}}\left(\Lambda^{\mathrm{eq}}, 0\right)$, i.e. $\widetilde{\mathscr{F}}^{\text {eq }}=\left.\widetilde{\mathscr{F}}\right|_{\left\{\Lambda^{\text {neq }}=(0, \ldots, 0)\right\}}$, and we let $\widetilde{\mathscr{F}}^{\text {neq }}=\widetilde{\mathscr{F}}-\widetilde{\mathscr{F}}^{\text {eq }}$. This leads to the splittings $[42]:$

$$
q=q^{\mathrm{eq}}+q^{\text {neq }}, \quad j=j^{\mathrm{eq}}+j^{\text {neq }},
$$

and

$$
\begin{array}{ll}
\sigma=\sigma^{\mathrm{eq}}+\sigma^{\text {neq }}, & H=H^{\mathrm{eq}}+H^{\text {neq }}, \\
m=m^{\mathrm{eq}}+m^{\text {neq }}, & M=M^{\mathrm{eq}}+M^{\text {neq }} .
\end{array}
$$

With slight abuse of notation we shall henceforth remove tildas and use the same symbol to denote a field and its associated response function.

Consistency with the dissipation inequality sets some restrictions on the response functions. One first such restriction is that the non-equilibrium part of the response functions associated to $\psi$ must vanish. Moreover, the constitutive equation for $\eta$ is determined by that of $\psi=\psi(\theta, F, \nabla F, \lambda, \nabla \lambda)$ :

$$
\eta=-\psi_{\theta}^{\prime}(\theta, F, \nabla F, \lambda, \nabla \lambda) .
$$

In addition, the equilibrium parts must satisfy:

$$
\begin{aligned}
q^{\mathrm{eq}} & =0, \quad j^{\mathrm{eq}}=0, \\
\sigma^{\mathrm{eq}} & =\psi_{F}^{\prime}(\theta, F, \nabla F, \lambda, \nabla \lambda), \\
H^{\mathrm{eq}} & =\psi_{\nabla F}^{\prime}(\theta, F, \nabla F, \lambda, \nabla \lambda), \\
m^{\mathrm{eq}} & =\psi_{\lambda}^{\prime}(\theta, F, \nabla F, \lambda, \nabla \lambda), \\
M^{\mathrm{eq}} & =\psi_{\nabla \lambda}^{\prime}(\theta, F, \nabla F, \lambda, \nabla \lambda) .
\end{aligned}
$$

As a consequence, (2.3) and (2.7) can be written, respectively, as

$$
\begin{aligned}
\theta \psi_{\theta \theta}^{\prime \prime}\left(\Lambda^{\mathrm{eq}}\right) \dot{\theta} & +\theta \psi_{\theta F}^{\prime \prime}\left(\Lambda^{\mathrm{eq}}\right): \dot{F}+\theta \psi_{\theta H}^{\prime}\left(\Lambda^{\mathrm{eq}}\right): \nabla \dot{F} \\
& +\theta \psi_{\theta \lambda}^{\prime \prime}\left(\Lambda^{\mathrm{eq}}\right) \cdot \dot{\lambda}+\theta \psi_{\theta \nabla \lambda}^{\prime \prime}\left(\Lambda^{\mathrm{eq}}\right): \nabla \dot{\lambda}+\operatorname{div} q+\mathscr{D}=0
\end{aligned}
$$

and

$$
0 \leq-\theta^{-1} q \cdot \nabla \theta+\mathscr{D}
$$

where $\mathscr{D}$ stands for the dissipation rate given here as

$$
\mathscr{D}:=-j \cdot \nabla \phi+\sigma^{\text {neq }}: \dot{F}+H^{\text {neq }}: \nabla \dot{F}+m^{\text {neq }} \cdot \dot{\lambda}+M^{\text {neq }}: \nabla \dot{\lambda} .
$$

Moreover, the heat flux $q$ and the electric curent $j$ can be given the form:

$$
q=-\mathbb{K}\left(\Lambda^{\mathrm{eq}}, \Lambda^{\mathrm{neq}}\right)\left[\Lambda^{\mathrm{neq}}\right], \quad j=-\mathbb{S}\left(\Lambda^{\mathrm{eq}}, \Lambda^{\mathrm{neq}}\right)\left[\Lambda^{\mathrm{neq}}\right],
$$

where $\mathbb{K}\left(\Lambda^{\mathrm{eq}}, \Lambda^{\text {neq }}\right)$ and $\mathbb{S}\left(\Lambda^{\mathrm{eq}}, \Lambda^{\text {neq }}\right)$ are linear maps and square brackets denote linear dependence. In particular, on this general level, we allow general cross effects such as Seebeck's and Peltier's effects (see e.g. [35, §3.2.2]), called also Thomson's thermoelectrical effect, although later we will restrict ourselves to much simplified case (3.11). The above set of restrictions follow from the application of a standard argument due to Coleman and Noll [11]. Strictly speaking, this argument requires the availability of source terms, the so-called virtual external microforces [2, §3.5], which support all conceivable processes by making it possible for the balance laws to be satisfied. In this paper we omit virtual microforces because anyhow they would be set to null in the system of partial differential equations we are going to derive. 
Consistency with the Second Law of Thermodynamics, as expressed by (2.14), still leaves ample freedom for the choice of the free energy and for the nonequilibrium parts of the constitutive response. Specific choices suitable for SMAs will be proposed in the next section. Since we want to account for rate-independent dissipation mechanisms which involve activation phenomena where evolution takes place only if the driving force exceeds some threshold (see also Example 3.2 below), we assume:

$$
m^{\text {neq }} \in \partial_{\dot{\lambda}} \xi_{0}\left(\Lambda^{\mathrm{eq}}, \dot{\lambda}, \nabla \dot{\lambda}\right)
$$

where $\xi_{0}$ is a pseudo-potential of dissipation, convex with respect to $\dot{\lambda}$ for each $\Lambda^{\text {eq }}$ and $\nabla \dot{\lambda}$ fixed, and possibly non smooth. Here $\partial$ is the subdifferential operator. For the remaining part of the non-equilibrium response, we consider:

$$
\begin{aligned}
\sigma^{\text {neq }} & =\left[\xi_{1}\right]_{\dot{F}}^{\prime}\left(\Lambda^{\mathrm{eq}}, \dot{F}, \nabla \dot{F}\right), \\
H^{\text {neq }} & =\left[\xi_{2}\right]_{\nabla \dot{F}}^{\prime}\left(\Lambda^{\mathrm{eq}}, \dot{F}, \nabla \dot{F}\right), \\
M^{\text {neq }} & =\left[\xi_{3}\right]_{\nabla \dot{\lambda}}^{\prime}\left(\Lambda^{\mathrm{eq}}, \dot{\lambda}, \nabla \dot{\lambda}\right),
\end{aligned}
$$

where $\xi_{1}, \xi_{2}$ and $\xi_{3}$ are smooth dissipation pseudopotentials, convex with respect to the variables $\dot{F}, \nabla \dot{F}$, and $\nabla \dot{\lambda}$, respectively. We require the pseudopotentials to have a minimum at 0 , that $\mathbb{S}\left(\Lambda^{\mathrm{eq}}, \Lambda^{\text {neq }}\right)\left[\Lambda^{\text {neq }}\right] \cdot \nabla \varphi \leq 0$, and that $\mathbb{K}\left(\Lambda^{\text {eq }}, \Lambda^{\text {neq }}\right)\left[\Lambda^{\text {neq }}\right] \cdot \nabla w \leq 0$ for all choices of $\Lambda^{\mathrm{eq}}$ and $\Lambda^{\text {neq }}$. The above assumption concerning $\mathbb{K}$ combined with the fact that $\mathscr{D} \geq 0$ provide sufficient conditions (although, in principle, not strictly necessary) which guarantee that (2.14) is satisfied. Using (2.12), (2.16), (2.17) and (2.18), equations (2.1)-(2.4) become:

$$
\begin{aligned}
& \varrho \ddot{y}-\operatorname{div}\left(\psi_{F}^{\prime}\left(\Lambda^{\mathrm{eq}}\right)+\left[\xi_{1}\right]_{\dot{F}}^{\prime}\left(\Lambda^{\mathrm{eq}}, \dot{F}, \nabla \dot{F}\right)\right. \\
& \left.+\operatorname{div}\left(\psi_{\nabla F}^{\prime}\left(\Lambda^{\mathrm{eq}}\right)+\left[\xi_{2}\right]_{\nabla \dot{F}}^{\prime}\left(\Lambda^{\mathrm{eq}}, \dot{F}, \nabla \dot{F}\right)\right)\right)=f, \\
& \partial_{\dot{\lambda}} \xi_{0}\left(\Lambda^{\mathrm{eq}}, \dot{\lambda}, \nabla \dot{\lambda}\right)+\psi_{\lambda}^{\prime}\left(\Lambda^{\mathrm{eq}}\right)-\operatorname{div}\left(\psi_{\nabla \lambda}^{\prime}\left(\Lambda^{\mathrm{eq}}\right)+\left[\xi_{3}\right]_{\nabla \dot{\lambda}}^{\prime}\left(\Lambda^{\mathrm{eq}}, \dot{\lambda}, \nabla \dot{\lambda}\right)\right) \ni 0, \\
& c_{\mathrm{v}}\left(\Lambda^{\mathrm{eq}}\right) \dot{\theta}+\operatorname{div}\left(\mathbb{K}\left(\Lambda^{\mathrm{eq}}, \Lambda^{\mathrm{neq}}\right)\left[\Lambda^{\mathrm{neq}}\right]\right) \\
& =\mathscr{D}+\psi_{\theta F}^{\prime \prime}\left(\Lambda^{\mathrm{eq}}\right): \dot{F}+\psi_{\theta \nabla F}^{\prime \prime}\left(\Lambda^{\mathrm{eq}}\right) \vdots \nabla \dot{F}+\psi_{\theta \lambda}^{\prime \prime}\left(\Lambda^{\mathrm{eq}}\right) \dot{\lambda}+\psi_{\theta \nabla \lambda}^{\prime \prime}\left(\Lambda^{\mathrm{eq}}\right) \cdot \nabla \dot{\lambda}, \\
& \operatorname{div}\left(\mathbb{S}\left(\Lambda^{\mathrm{eq}}, \Lambda^{\text {neq }}\right)\left[\Lambda^{\text {neq }}\right]\right)=0,
\end{aligned}
$$

where

is the heat capacity.

$$
c_{\mathrm{v}}\left(\Lambda^{\mathrm{eq}}\right)=\theta \psi_{\theta \theta}^{\prime \prime}\left(\Lambda^{\mathrm{eq}}\right)
$$

\section{A special case of electrically conductive SMAs}

We shall restrict attention to a special case that makes a compromise still well fitted with describing main phenomena of electrically conductive shape-memory alloys as mentioned in Section 1 and allowing for rigorous analysis outlined in the following Section 4. It will be convenient, from the mathematical point of view, to introduce an "enthalpy" $w=h(\theta)$ with $h(\cdot)$ defined from (3.5), and use it as the unknown in place of temperature and complete it by boundary conditions in order to obtain a new system which is better suited for mathematical analysis.

Before further specializing our constitutive equations, we want to point out some simplifications that will be made. The most important one concerns the choice of the dissipation functionals (3.10) for the stress and the hyperstress. Specifically, we will choose for $\sigma^{\text {neq }}$ and $H^{\text {neq }}$ pseudopotentials that are "frame-indifferent only 
up to infinitesimally small rotations". We will also rule out general coupling crosseffects between thermal and electric flows (2.16) and confine ourselves to standard Fourier's and Ohm's laws (3.11). We further make a modeling assumption that the free energy is "partly decoupled" and linearized around some reference temperature, namely:

$$
\psi=\varphi_{0}(F, \lambda)+\frac{1}{2} \nu|\nabla F|^{2}+\frac{1}{2} \beta|\nabla \lambda|^{2}+\theta \varphi_{1}(F)-\varphi_{2}(\theta),
$$

where $\varphi_{0}: \mathbb{R}^{3 \times 3} \times \mathbb{R}^{L} \rightarrow \mathbb{R}, \varphi_{1}: \mathbb{R}^{3 \times 3} \rightarrow \mathbb{R}$, and $\varphi_{2}: \mathbb{R} \rightarrow \mathbb{R}$ are $C^{1}$-functions, and where $\nu>0$ and $\beta \geq 0$. Additional data qualifications will be specified later. Both $\varphi_{0}$ and $\varphi_{1}$ will be assumed frame indifferent, i.e. $\varphi_{0}(R F, \lambda)=\varphi_{0}(F, \lambda)$ and $\varphi_{1}(R F)=\varphi_{1}(F)$ for any $R \in \mathrm{SO}(3)$, the special orthogonal group of $3 \times 3$-matrices.

By (3.1), the last four equations of (2.12) become

$$
\begin{aligned}
\sigma^{\mathrm{eq}} & =\left[\varphi_{0}\right]_{F}^{\prime}(F, \lambda)+\theta \varphi_{1}^{\prime}(F), & & H^{\mathrm{eq}}=\nu \nabla^{2} y, \\
m^{\mathrm{eq}} & =\left[\varphi_{0}\right]_{\lambda}^{\prime}(F, \lambda), & & M^{\mathrm{eq}}=\beta \nabla \lambda,
\end{aligned}
$$

and (2.13) becomes

$$
c_{\mathrm{V}}(\theta) \dot{\theta}=-\operatorname{div} q+\theta \varphi_{1}^{\prime}(F): \dot{F}+\mathscr{D} .
$$

Note that the internal energy splits additively into a part that depends on $F, \nabla F$, $\lambda$, and $\nabla \lambda$ but not on $\theta$, and a part that depends on $\theta$ only. In fact, from (3.1) and (2.11) we obtain $\eta=-\varphi_{1}(F)+\varphi_{2}^{\prime}(\theta)$ and, recalling (2.7),

$$
\varepsilon=\varphi_{0}(F, \lambda)+\frac{1}{2} \nu|\nabla F|^{2}+\frac{1}{2} \beta|\nabla \lambda|^{2}+h(\theta),
$$

where

$$
h(\theta):=-\varphi_{2}(\theta)+\theta \varphi_{2}^{\prime}(\theta) .
$$

The splitting (3.4) is also found in [2], where our "enthalpy" $h(\theta)$ is referred to as "thermal energy". Observe that from (3.5) we have $h^{\prime}(\theta)=\theta \varphi_{2}^{\prime \prime}(\theta)=c_{\mathrm{v}}(\theta)$, hence (3.3) may be written as:

$$
(h(\theta))^{\bullet}=-\operatorname{div} q+\theta \varphi_{1}^{\prime}(F): \dot{F}+\mathscr{D} .
$$

This suggests to replace the unknown $\theta$ with $w=h(\theta)$. This "enthalpy transformation" is possible by making the additional assumption that the function $h: \mathbb{R}^{+} \rightarrow \mathbb{R}^{+}$is invertible. This allows us to express $\theta$ in terms of $w$ by writing

$$
\theta=\mathscr{T}(w):= \begin{cases}h^{-1}(w) & \text { if } w \geq 0 \\ 0 & \text { if } w=0\end{cases}
$$

so that (3.6) becomes

$$
\dot{w}=-\operatorname{div} q+\mathscr{T}(w) \varphi_{1}^{\prime}(F): \dot{F}+\mathscr{D},
$$

which we refer to as "enthalpy equation". While the choice of $\psi$ determines the equilibrium part of the response functions, the non-equilibrium part must still be postulated. To model the dissipation in SMAs we make the following choices for the pseudopotentials introduced in (2.17)-(2.18):

$$
\begin{aligned}
\xi_{0}\left(\Lambda^{\text {eq }}, \dot{\lambda}, \nabla \dot{\lambda}\right) & =\zeta(\dot{\lambda}), \\
\xi_{1}\left(\Lambda^{\mathrm{eq}}, \dot{F}, \nabla \dot{F}\right) & =\frac{1}{2} \mathbb{D}(\operatorname{sym} \dot{F}):(\operatorname{sym} \dot{F}), \\
\xi_{2}\left(\Lambda^{\mathrm{eq}}, \dot{F}, \nabla \dot{F}\right) & =\frac{1}{2} \mu|\nabla \dot{F}|^{2} \\
\xi_{3}\left(\Lambda^{\text {eq }}, \dot{\lambda}, \nabla \dot{\lambda}\right) & =0 .
\end{aligned}
$$


Here $\zeta: \mathbb{R}^{L} \rightarrow \mathbb{R}^{+}$is a non-negative convex function and $\mathbb{D}$ is a positive-definite fourth-order tensor whose components $\mathbb{D}_{i j k l}$ satisfy $\mathbb{D}_{i j k l}=\mathbb{D}_{j i k l}=\mathbb{D}_{i j l k}$. Combining (2.18) with (3.9) and recalling that $\operatorname{sym}(\dot{F})=e(\dot{y})$, we obtain

$$
\begin{aligned}
\sigma^{\text {neq }} & =\mathbb{D}(e(\dot{y})), \\
H^{\text {neq }} & =\mu \nabla \dot{F}, \\
m^{\text {neq }} & \in \partial \zeta(\dot{\lambda}), \\
M^{\text {neq }} & =0 .
\end{aligned}
$$

We assume that the material heat flux $q$ and the material flux of electric current $j$ have the form

$$
q=-\mathbb{K}(\theta, F, \lambda) \nabla \theta, \quad j=-\mathbb{S}(\theta, F, \lambda) \nabla \phi,
$$

with $\mathbb{K}$ and $\mathbb{S}$ positive definite matrices of heat conductivity and electric conductivity, respectively. Naturally, both $\mathbb{K}$ and $\mathbb{S}$ are to be frame indifferent, i.e. $\mathbb{K}(\theta, R F, \lambda)=$ $\mathbb{K}(\theta, F, \lambda)$ and $\mathbb{S}(\theta, R F, \lambda)=\mathbb{S}(\theta, F, \lambda)$ for any $R \in \mathrm{SO}(3)$. By adopting (3.11) we rule out the variety of coupling phenomena that, in principle, would be compatible with the general representation (2.16) but would produce difficulties in the mathematical analysis. The first constitutive equation in (3.11) is the Fourier law while the second one is the Ohm law. Note that both $\mathbb{K}$ and $\mathbb{S}$ are allowed to depend, beside $\theta$ as quite usual in nonlinear media, also on $F$ and/or $\lambda$, which allows to keep track particular phases: e.g. the order parameter $\lambda$ is a natural quantity to distinguish conductivity in austenite and martensite. Alternatively, one can also employ the right Cauchy-Green tensor $F^{\top} F$ which is typically close to $\mathbb{I}$ in austenite while in martensite it is substantially different. In a very special case of R-phase in NiTi, electric conductivity is assumed to depend on the angle of the atomic lattice which may vary quite continuously so that a wide range of $F^{\top} F$ is to be taken into account for the dependence of $\mathbb{S}$ on $F$.

The next step is to express the conductivities in terms of $w$. This is easily done:

$$
\mathscr{K}(w, F, \lambda):=\frac{\mathbb{K}(\mathscr{T}(w), F, \lambda)}{c_{\mathrm{v}}(\mathscr{T}(w))}, \quad \mathscr{S}(w, F, z):=\mathbb{S}(\mathscr{T}(w), F, \lambda)
$$

It is important that the enthalpy transformation preserves the linear dependence of the heat flux on the gradient of the thermal variable, namely $q=-\mathscr{K}(w, F, \lambda) \nabla w$. In view of (2.10), (2.18) and (3.10), the balance of linear momentum (2.1) and the balance of microforces (2.2), the enthalpy equation (3.8), and the Kirchhoff law for electric current (2.4) are transformed into the following system:

$$
\begin{aligned}
& \varrho \ddot{y}-\operatorname{div}\left(\left[\varphi_{0}\right]_{F}^{\prime}(\nabla y, \lambda)+\mathscr{T}(w) \varphi_{1}^{\prime}(\nabla y)+\mathbb{D} e(\dot{y})-\operatorname{div} \nabla^{2}(\nu y+\mu \dot{y})\right)=f, \\
& \partial \zeta(\dot{\lambda})+\left[\varphi_{0}\right]_{\lambda}^{\prime}(\nabla y, \lambda)-\beta \Delta \lambda \ni 0 \\
& \dot{w}-\operatorname{div}(\mathscr{K}(w, \nabla y, \lambda) \nabla w)=\zeta(\dot{\lambda})+\mathbb{D} e(\dot{y}): e(\dot{y})+\mu\left|\nabla^{2} \dot{y}\right|^{2} \\
& +\mathscr{S}(w, \nabla y, \lambda) \nabla \phi \cdot \nabla \phi+\mathscr{T}(w) \varphi_{1}^{\prime}(\nabla y): \nabla \dot{y}, \\
& \operatorname{div}(\mathscr{S}(w, \nabla y, \lambda) \nabla \phi)=0 .
\end{aligned}
$$

The initial-value problem we consider is obtained by augmenting (3.13) with the following initial conditions:

$$
y(0)=y_{0}, \quad \dot{y}(0)=\dot{y}_{0}, \quad \lambda(0)=\lambda_{0}, \quad w(0)=w_{0} .
$$

We may complete the system (3.13) by boundary conditions which may combine various mechanical, thermal, and electric loadings. For simplicity, let us consider only two kinds of boundary conditions and thus $\partial \Omega$ divided as

$$
\partial \Omega=\Gamma_{0} \cup \Gamma_{1} \cup N, \quad \Gamma_{0} \cap \Gamma_{1}=\emptyset,
$$


with $\Gamma_{0}$ and $\Gamma_{1}$ relatively open in $\partial \Omega$, and $\operatorname{meas}_{2}(N)=0$. Then we select, for the mechanical and the micromechanical parts:

$$
\left\{\begin{array}{ll}
(\sigma-\operatorname{div} H) \mathbf{n}=g, & \text { on } \Gamma_{1} \\
\left.y\right|_{\Gamma_{0}}=y_{\mathrm{D}} & \text { on } \Gamma_{0},
\end{array} \quad H \mathbf{n}=0 \quad \text { and } \quad M \mathbf{n}=0 \quad \text { on } \partial \Omega,\right.
$$

where $g: \Gamma_{1} \rightarrow \mathbb{R}^{3}$ is a prescribed surface force. In our case, it means

$$
\begin{cases}\left(\left[\varphi_{0}\right]_{F}^{\prime}(\nabla y, \lambda)+\mathbb{D}(e(\dot{y}))-\operatorname{div} \nabla^{2}(\nu y+\mu \dot{y})\right) \mathbf{n}=g, & \text { on } \Gamma_{1} \\ \left.y\right|_{\Gamma_{0}}=y_{\mathrm{D}} & \text { on } \Gamma_{0},\end{cases}
$$

and

$$
\frac{\partial \nabla y}{\partial \mathbf{n}}=0 \quad \text { and } \quad \beta \frac{\partial \lambda}{\partial \mathbf{n}}=0 \quad \text { on } \partial \Omega .
$$

For the external heat flux $h_{\mathrm{ext}}$ and the electric currents we choose

$$
\mathscr{K}(w, \nabla y, \lambda) \nabla w \cdot \mathbf{n}=h_{\text {ext }} \text { on } \partial \Omega, \quad \begin{cases}\mathscr{S}(w, \nabla y, \lambda) \nabla \phi \cdot \mathbf{n}=0 & \text { on } \Gamma_{1} \\ \left.\phi\right|_{\Gamma_{0}}=\phi_{\text {ext }}, & \text { on } \Gamma_{0},\end{cases}
$$

so that (particular components of) $\Gamma_{0}$ has a meaning of electrodes with prescribed electric potential and mechanical fixing, while $\Gamma_{1}$ is a part which is electrically isolated. Such conditions correspond rather to an ideal situation when, e.g., an SMA wire is plugged in a hard mechanical device with an infinitesimally hard source of electric voltage on infinitesimally conductive electrodes. Of course, in case of more realistic conditions, we would consider rather conditions of a (nonlocal) Robin type. Also, time-dependent Dirichlet condition $y_{\mathrm{D}}$ which is of a practical interest will be simplified in Section 4.

Example 3.1. (Free energy (3.1) in SMAs.) We consider $L-1$ variants of martensite which are standardly determined, in the stress-free state, by distortion matrices $U_{\ell}$, $\ell=2, \ldots, L$, while the cubic austenite corresponds to $U_{1}=\mathbb{I}$. Further we define the mapping $\mathscr{L}: \mathbb{R}^{3 \times 3} \rightarrow \mathbb{R}^{L}$ that identifies particular variants, namely we assume that $\mathscr{L}$ is frame indifferent, $\mathscr{L}_{\ell} \geq 0$ and $\mathscr{L}_{\ell}(F)=1$ if $F^{\top} F$ is near $U_{\ell}^{\top} U_{\ell}$ for all $\ell=1, \ldots, L$ and $\sum_{\ell=1}^{L} \mathscr{L}_{\ell}=1$. Then, using the concept of St.Venant-Kirchhoff material for each particular phase variant (cf. [40, Sect.6.6] or also [26]), we postulate

$$
\begin{array}{r}
\varphi_{0}(F, \lambda):=\min _{\ell=1, \ldots, L} \frac{1}{2} \sum_{i, j, k, l=1}^{d} \varepsilon_{i j}^{\ell} \mathbb{C}_{i j k l}^{\ell} \varepsilon_{k l}^{\ell}+K|\lambda-\mathscr{L}(F)|^{2}-k_{\mathrm{cc}} \theta_{\mathrm{R}} \mathscr{L}_{1}(F) \\
\text { with } \varepsilon^{\ell}=\frac{R_{\ell}^{\top}\left(U_{\ell}^{\top}\right)^{-1} F^{\top} F U_{\ell}^{-1} R_{\ell}-\mathbb{I}}{2}
\end{array}
$$

$\varphi_{1}(F):=k_{\mathrm{cc}} \mathscr{L}_{1}(F)$,

where $\mathbb{C}^{\ell}=\left\{\mathbb{C}_{i j k l}^{\ell}\right\}$ is the 4 th-order tensor of elastic moduli satisfying the usual symmetry relations depending also on symmetry of the specific phase (variant) $\ell$, $R_{\ell}$ is a rotation matrix transforming the basis of the austenite to the basis of the martensitic variant $\ell$ (in particular $R_{1}=\mathbb{I}$ ). Further, $k_{\mathrm{cc}}$ is the so-called ClausiusClapeyron constant multiplied by a transformation strain (in $\mathrm{Pa} / \mathrm{K}$ ), and $\theta_{\mathrm{R}}$ is the equilibrium temperature under which austenite and martensite has equal energy in stress-free space. Strictly speaking, the vectorial order parameter $\lambda$ cannot be given the meaning of relative volume fraction of the variants like in [16] because here we do not enforce any constraints of the form $0 \leq \lambda_{\ell} \leq 1$ and $\sum_{\ell=1}^{L} \lambda_{\ell}=1$. However, the constant $K$ should be chosen large, so that presumably $\lambda$ will mostly be close to $\mathscr{L}(\nabla y)$ in $\mathbb{R}^{L}$, and hence approximately $(0, \ldots, 1, \ldots, 0)$ on domains occupied by any particular pure phase variants. 
Note that (3.20a) complies with (4.2q) below. Due to usage of "min" in (3.20a), $\varphi_{0}$ is nonsmooth hence certain smoothening is to be theoretical made to comply with the smooth ansatz (2.12). For more sophisticated construction method of $\varphi_{0}$ and $\varphi_{1}$ based on cubic $C^{2}$-splines fitted with experimentally-measured wells and elastic moduli in specific shape-memory materials we refer to [23].

Example 3.2. (Dissipation of mechanical energy in SMAs.) The analysis of a isothermal model with capillarity energy carried out in [43] shows that if dissipation mechanisms are of viscous type, then dissipation vanishes in the limit of infinitely-slow loading. This suggests that models embodying viscous-like dissipation cannot entirely properly reproduce the hysteretic behavior displayed by shape-memory alloys as a phenomenon partly independent from the elastic response. To account for such behavior, it seems reasonable to incorporate some rate-independent dissipation in the model through the pseudopotential of dissipation $\zeta: \mathbb{R}^{L} \rightarrow \mathbb{R}^{+}$positively homogeneous of degree-1, as already suggested in [45] and, for the isothermal case, analyzed and used in [4, 3, 39]. By a standard result from Convex Analysis [44, Corollary 13.2.2] the pseudopotential of dissipation $\zeta: \mathbb{R}^{L} \rightarrow \mathbb{R}^{+}$introduced in (3.9a) is the Legendre-Fenchel conjugate of $\delta_{K}$, the indicator function of a closed, nonempty bounded convex set $K \subset \mathbb{R}^{L}$. By another standard result [44, Corollary 23.5.1], $\partial \zeta=\left(\partial \delta_{K}\right)^{-1}$, that is to say, $m \in \partial \zeta(\dot{\lambda})$ if and only if $\dot{\lambda} \in \partial \delta_{K}(m)$. In particular, equation (3.13b) states that the following two conditions must hold at each time $t \in(0, T)$, and at each point $x \in \Omega$ : first, the driving microforce $m^{\mathrm{dr}}(t, x):=\beta \Delta \lambda(t, x)-\left[\varphi_{0}\right]_{\lambda}^{\prime}(\nabla y(t, x), \lambda(t, x))$ must be confined in $K$; second, phase transformation (i.e. $\dot{\zeta} \neq 0$ ) takes place only if the driving microforce is on the boundary of $K$. Thus, the boundary of $K$ determines the activation threshold for phase transformation. Note that the homogeneity of $\zeta$, along with its non-negativity, implies $0 \in \partial \zeta(0)$, therefore $0 \in \partial \delta_{K}(0)$ and $K$ contains the null vector.

\section{Analysis of the SMA model in brief}

The rigorous mathematical analysis of the initial-boundary-value problem (3.14)(3.17)-(3.18)-(3.19) for the system (3.13) as far as mere existence of a (suitably defined) solution is rather complicated and requires a fine combination of theory of rate-independent processes by Mielke at al. [15, 27, 29, 30] adapted for coupling with viscous/inertial effects [49] and sophisticated estimates by Boccardo and Gallouët $[7,8]$ of the temperature gradient of the heat-equation with $L^{1}$ - or even measure data. This general scenario has essentially been done in a general-purpose paper [50] in the context of small strains and convex energies without $\nabla F$-terms and Joule-heating coupling, extending the isothermal situation scrutinzed in [49]. The particular modifications for the specific model like (3.13) have been only proposed in [50, Example 5.6 with Remarks 4.8, 4.9, 5.7]. Therefore, we will present the systematic analysis to some (though not much deep) details here. For simplicity, we consider a smooth initial condition $y_{0}$, a time-independent hard-device loading $y_{\mathrm{D}}$, and a vanishing surface load $g=0$, cf. Remark 4.4 below for more general loads.

Let us abbreviate $I:=(0, T), Q:=I \times \Omega, \Sigma:=I \times \partial \Omega$. We will use standard notation for function space, namely spaces of continuous $\mathbb{R}^{k}$-valued functions $C\left(\bar{\Omega} ; \mathbb{R}^{k}\right)$, continuously differentiable functions $C^{1}\left(\bar{\Omega} ; \mathbb{R}^{k}\right)$, Lebesgue spaces $L^{p}\left(\Omega ; \mathbb{R}^{k}\right)$ and Sobolev spaces $W^{1, p}\left(\Omega ; \mathbb{R}^{k}\right)$, Bochner spaces of $X$-valued functions $L^{p}(I ; X)$. Moreover, we denote by $\mathrm{B}(\bar{I} ; X), \mathrm{BV}(\bar{I} ; X)$, or $C_{\mathrm{w}}(\bar{I} ; X)$ the Banach space of the functions $\bar{I} \rightarrow X$ that are bounded measurable, have a bounded variation, or are weakly continuous, respectively; note that all these functions are defined everywhere on $\bar{I}$. We will use the notation $p^{\prime}=p /(p-1)$ for the conjugate exponent to $p$. Instead of $y(t, \cdot)$ or $\lambda(t, \cdot)$ or $w(t, \cdot)$, we will write briefly $y(t)$ or $\lambda(t)$ or $w(t)$, respectively. 
We have to make various data qualifications, some of them physically natural, the others physically not much restricted and just for mathematical techniques used below (in some case, even weaker assumptions work under more advanced techniques [50]). As we admit both gradient- and no-gradient theory for $\lambda$ depending on $\beta$ from (3.1), we abbreviate

$$
V:= \begin{cases}W^{1,2}\left(\Omega ; \mathbb{R}^{L}\right) & \text { if } \beta>0 \\ L^{2}\left(\Omega ; \mathbb{R}^{L}\right) & \text { if } \beta=0 .\end{cases}
$$

Then, we make the following assumptions:

$\Omega$ is a bounded Lipschitz domain in $\mathbb{R}^{3}, \operatorname{meas}_{2}\left(\Gamma_{0}\right) \neq 0$,

$\varrho>0, \mu>0, \nu>0, \beta \geq 0$,

$\exists \alpha \geq 0: \quad(F, \lambda) \mapsto \varphi_{0}(F, \lambda)+\alpha|F|^{2}$ is strictly convex,

$\exists c_{\mathbb{D}}>0 \forall e \in \mathbb{R}^{3 \times 3}: \quad \mathbb{D} e: e \geq c_{\mathbb{D}}|e|^{2}$,

$\varphi_{0}: \mathbb{R}^{3 \times 3} \times \mathbb{R}^{L} \rightarrow \mathbb{R}^{+}, \varphi_{1}: \mathbb{R}^{3 \times 3} \rightarrow \mathbb{R}^{+}$are continuously differentiable,

$\exists q_{0}>1, \alpha_{0}>0 \forall(F, \lambda) \in \mathbb{R}^{3 \times 3} \times \mathbb{R}^{L}: \quad \varphi_{0}(F, \lambda) \geq \alpha_{0}|\lambda|^{q_{0}}$,

$\left|\varphi_{0}(F, \lambda)\right| \leq C\left(1+|F|^{6-\epsilon}+|\lambda|^{b}\right)$ with $b=2$ (if $\left.\beta=0\right)$ or $b<6$ (if $\left.\beta>0\right)$,

$\left|\left[\varphi_{0}\right]_{F}^{\prime}(F, \lambda)\right| \leq C\left(1+|F|^{5}+|\lambda|\right)$,

$\exists \ell \in \mathbb{R}: \quad\left|\left[\varphi_{0}\right]_{F}^{\prime}(F, \lambda)-\left[\varphi_{0}\right]_{F}^{\prime}(\tilde{F}, \lambda)\right| \leq \ell\left(1+|F|^{4}+|\tilde{F}|^{4}+|\lambda|\right)|F-\tilde{F}|$,

$\varphi_{1}^{\prime}$ is bounded,

$c_{\mathrm{v}}: \mathbb{R} \rightarrow \mathbb{R}^{+}$continuous, and

$$
\exists \omega_{1} \geq \omega>\frac{6}{5}, \quad c_{1} \geq c_{0}>0 \forall \theta \in \mathbb{R}^{+}: c_{0}(1+\theta)^{\omega-1} \leq c_{\mathrm{v}}(\theta) \leq c_{1}(1+\theta)^{\omega_{1}-1},
$$

$\mathscr{S}: \mathbb{R} \times \mathbb{R}^{3 \times 3} \times \mathbb{R}^{L} \rightarrow \mathbb{R}^{3 \times 3}$ is bounded, continuous, and

$$
\exists \alpha_{\mathbb{S}} \geq 0 \forall(\theta, F, \lambda, g) \in \mathbb{R} \times \mathbb{R}^{3 \times 3} \times \mathbb{R}^{L} \times \mathbb{R}^{3}: \quad \mathscr{S}(\theta, F, \lambda) g \cdot g \geq \alpha_{\mathbb{S}},
$$

$\mathscr{K}: \mathbb{R} \times \mathbb{R}^{3 \times 3} \times \mathbb{R}^{L} \rightarrow \mathbb{R}^{3 \times 3}$ is bounded, continuous, and

$$
\exists \alpha_{\mathbb{K}} \geq 0 \forall(\theta, F, \lambda, g) \in \mathbb{R} \times \mathbb{R}^{3 \times 3} \times \mathbb{R}^{L} \times \mathbb{R}^{3}: \quad \mathscr{K}(\theta, F, \lambda) g \cdot g \geq \alpha_{\mathbb{K}},
$$

$y_{0} \in W^{2, \gamma}\left(\Omega ; \mathbb{R}^{3}\right), \gamma>\frac{6 \omega q_{0}}{\omega q_{0}-5}, \quad \dot{y}_{0} \in L^{2}\left(\Omega ; \mathbb{R}^{3}\right), \quad \theta_{0} \in L^{\omega}(\Omega), \quad \theta_{0} \geq 0, \quad \lambda_{0} \in V$,

$\forall \tilde{\lambda} \in V: \quad \int_{\Omega} \varphi_{0}\left(\nabla y_{0}, \lambda_{0}\right) \mathrm{d} x \leq \int_{\Omega} \varphi_{0}\left(\nabla y_{0}, \tilde{\lambda}\right)+\zeta\left(\tilde{\lambda}-\lambda_{0}\right) \mathrm{d} x$,

$f \in L^{1}\left(I ; L^{2}\left(\Omega ; \mathbb{R}^{3}\right)\right), \quad y_{\mathrm{D}} \in W^{2-1 / \gamma, \gamma}\left(\Gamma_{0} ; \mathbb{R}^{3}\right)$,

$h_{\mathrm{ext}} \in L^{1}(\Sigma), \quad h_{\mathrm{ext}} \geq 0, \quad \phi_{\mathrm{ext}} \in L^{2}\left(I ; W^{1 / 2,2}\left(\Gamma_{0}\right)\right)$,

if $\beta=0$, then $\mathbb{S}(\theta, F, \cdot)$ and $\mathbb{K}(\theta, F, \cdot)$ are constant, and

$$
\varphi_{0}(F, \lambda)=\varphi_{00}(F)+\varphi_{01}(F) \cdot \lambda+\varphi_{02}(\lambda) \text { with } \varphi_{02} \text { quadratic } .
$$

Let us note that (3.20) satisfies (4.2) with $q_{0}=2$ under the mild assumption that all $\mathbb{C}^{\ell}$ are positive definite and $\mathscr{L}$ together with $\mathscr{L}^{\prime}$ are is Lipschitz continuous; here it is important that $(F, \lambda) \mapsto|\lambda|^{2}-\lambda \cdot \mathscr{L}(F)$ complies with (4.2c). Also $\beta=0$ is allowed if $\mathscr{S}$ and $\mathscr{K}$ do not depend on $\lambda$ because $\varphi_{0}$ from (3.20a) complies with $(4.2 \mathrm{q})$. Also note that $(4.2 \mathrm{l}, \mathrm{m})$ together with $(4.2 \mathrm{k})$ represent, in fact, qualification of $\mathbb{S}$ and $\mathbb{K}$ through (3.12).

The theory of rate-independent processes routinely employs time semidiscretization with a constant time step $\tau$ to construct an auxiliary approximated solution, then proves suitable a-priori estimates, and passes $\tau \rightarrow 0$. Here, employing still some auxiliary regularization to compensate the superlinear growth of the heat-source terms in $(3.13 \mathrm{c})$, it leads to the recursive formula for the quadruple 
$\left(y_{\tau}^{k}, \lambda_{\tau}^{k}, \theta_{\tau}^{k}, \phi_{\tau}^{k}\right)$ solving the system

$$
\begin{gathered}
\varrho \frac{y_{\tau}^{k}-2 y_{\tau}^{k-1}+y_{\tau}^{k-2}}{\tau^{2}}-\operatorname{div}\left(\sigma_{\tau}^{k}-\operatorname{div} H_{\tau}^{k}\right)=f_{\tau}^{k} \quad \text { with } \\
\sigma_{\tau}^{k}:=\left[\varphi_{0}\right]_{F}^{\prime}\left(\nabla y_{\tau}^{k}, \lambda_{\tau}^{k}\right)+\mathscr{T}\left(w_{\tau}^{k}\right) \varphi_{1}^{\prime}\left(\nabla y_{\tau}^{k}\right)+\mathbb{D} e\left(\frac{y_{\tau}^{k}-y_{\tau}^{k-1}}{\tau}\right) \quad \text { and } \\
H_{\tau}^{k}:=\mu \Delta \nabla \frac{y_{\tau}^{k}-y_{\tau}^{k-1}}{\tau}+\operatorname{div}\left(\left(\nu+\tau\left|\nabla^{2} y_{\tau}^{k}\right|^{\gamma-2}\right) \nabla^{2} y_{\tau}^{k}\right) \\
\partial \zeta\left(\frac{\lambda_{\tau}^{k}-\lambda_{\tau}^{k-1}}{\tau}\right)+\left[\varphi_{0}\right]_{\lambda}^{\prime}\left(\nabla y_{\tau}^{k}, \lambda_{\tau}^{k}\right)-\beta \Delta \lambda_{\tau}^{k} \ni 0 \\
\frac{w_{\tau}^{k}-w_{\tau}^{k-1}}{\tau}-\operatorname{div}\left(\mathscr{K}\left(w_{\tau}^{k}, \nabla y_{\tau}^{k}, \lambda_{\tau}^{k}\right) \nabla w_{\tau}^{k}\right)=\zeta\left(\frac{\lambda_{\tau}^{k}-\lambda_{\tau}^{k-1}}{\tau}\right) \\
+(1-\sqrt{\tau}) \mathbb{D} e\left(\frac{y_{\tau}^{k}-y_{\tau}^{k-1}}{\tau}\right): e\left(\frac{y_{\tau}^{k}-y_{\tau}^{k-1}}{\tau}\right)+(1-\sqrt{\tau}) \mu\left|\nabla^{2} \frac{y_{\tau}^{k}-y_{\tau}^{k-1}}{\tau}\right|^{2} \\
\quad+\mathscr{S}\left(w_{\tau}^{k}, \nabla y_{\tau}^{k}, \lambda_{\tau}^{k}\right) \nabla \phi_{\tau}^{k} \cdot \nabla \phi_{\tau}^{k}+\mathscr{T}\left(w_{\tau}^{k}\right) \varphi_{1}^{\prime}\left(\nabla y_{\tau}^{k}\right): \nabla \frac{y_{\tau}^{k}-y_{\tau}^{k-1}}{\tau},
\end{gathered}
$$

for $k=1, \ldots, K_{\tau}:=T / \tau$, starting from $k=1$ by using

$$
y_{\tau}^{0}=y_{0}, \quad y_{\tau}^{-1}=y_{0}-\tau \dot{y}_{0}, \quad \lambda(0)=\lambda_{\tau}^{0}, \quad w_{\tau}^{0}=\mathscr{T}\left(\theta_{0}\right),
$$

completed with the corresponding boundary conditions

$$
\begin{array}{lll}
\left(\sigma_{\tau}^{k}-\operatorname{div} H_{\tau}^{k}\right) \mathbf{n}=0, & \mathscr{S}\left(w_{\tau}^{k}, \nabla y_{\tau}^{k}, \lambda_{\tau}^{k}\right) \nabla \phi_{\tau}^{k} \cdot \mathbf{n}=0 & \text { on } \Gamma_{1}, \\
y_{\tau}^{k}=y_{\mathrm{D}}, & \left.\phi_{\tau}^{k}\right|_{\Gamma_{0}}=\phi_{\mathrm{ext}, \tau}^{k} & \text { on } \Gamma_{2}, \\
\frac{\partial \nabla y_{\tau}^{k}}{\partial \mathbf{n}}=0, \quad \beta \frac{\partial \lambda_{\tau}^{k}}{\partial \mathbf{n}}=0, & \mathscr{K}\left(w_{\tau}^{k}, \nabla y_{\tau}^{k}, \lambda_{\tau}^{k}\right) \nabla w_{\tau}^{k} \cdot \mathbf{n}=h_{\mathrm{ext}, \tau}^{k} & \text { on } \partial \Omega,
\end{array}
$$

where the discrete stress $\sigma_{\tau}^{k}$ and hyperstress $H_{\tau}^{k}$ in (4.5a) are from (4.3a), and where $f_{\tau}^{k}$ in (4.3a) and $h_{\mathrm{ext}, \tau}^{k}$ and $\phi_{\mathrm{ext}, \tau}^{k}$ in $(4.5 \mathrm{~b}, \mathrm{c})$ approximate $f, \phi_{\mathrm{ext}}$, and $h_{\mathrm{ext}}$ at $t=k \tau$, respectively, cf. (4.15) below.

Existence of a (not necessarily unique) standard weak solution $\left(y_{\tau}^{k}, \lambda_{\tau}^{k}, w_{\tau}^{k}, \phi_{\tau}^{k}\right) \in W^{2, \gamma}\left(\Omega ; \mathbb{R}^{3}\right) \times V \times W^{1,2}(\Omega) \times W^{1,2}(\Omega)$ to the boundaryvalue problem (4.5) for the system (4.3) can be shown by usual monotonicity methods, coercivity arguments, and compact embeddings provided the exponent $\gamma$ in the regularizing term is sufficiently large as specified in $(4.2 \mathrm{n})$; it allows us to execute estimates by testing (4.3) subsequently by $y_{\tau}^{k}, \lambda_{\tau}^{k},\left|w_{\tau}^{k}\right|^{\alpha-1} w_{\tau}^{k}$, and $\phi_{\tau}^{k}$, with $0<\alpha<q_{0}-1$ to show coercivity of the underlying nonlinear operator governing (4.3). It is also important that $w_{\tau}^{k} \geq 0$ can be shown by testing (4.3c) by the negative part of $w_{\tau}^{k}$ which again belongs to $W^{1,2}(\Omega)$ and is, therefore, the legal test function.

One of the very essential tricks is that we assume $\tau>0$ small enough, namely $\tau<\tau_{0}$ with $\tau_{0}$ such that

$$
\forall y \in W^{2,2}\left(\Omega ; \mathbb{R}^{3}\right),\left.y\right|_{\Gamma_{0}}=0: \quad \int_{\Omega} \frac{c_{\mathbb{D}}}{\sqrt{\tau_{0}}}|e(y)|^{2}-\alpha|\nabla y|^{2} \mathrm{~d} x \geq 0
$$

with $\alpha$ from (4.2c) and with $c_{\mathbb{D}}$ from (4.2d). Then, by using (4.2c) and (4.6) together with Korn's inequality relying on non-trivial Dirichlet boundary conditions $(4.2 \mathrm{a})$, we can see that the functional $(y, \lambda) \mapsto \int_{\Omega} \frac{1}{\tau} \mathbb{D} e(y): e(y)+\varphi_{0}(y, \lambda) \mathrm{d} x$ is strictly convex for $\tau<\tau_{0}$. For some chosen (but fixed) $y_{\tau}^{k}$ and $w_{\tau}^{k}$, we still consider a 
modified minimization problem, namely

$$
\begin{aligned}
\operatorname{minimize} & \int_{\Omega} \varrho \frac{y_{\tau}^{k}-2 y_{\tau}^{k-1}+y_{\tau}^{k-2}}{\tau^{2}} \cdot y+\tau \zeta\left(\frac{\lambda-\lambda_{\tau}^{k-1}}{\tau}\right)+\frac{\tau}{\gamma}\left|\nabla^{2} y_{\tau}^{k}\right|^{\gamma}-f_{\tau}^{k} \cdot y \\
& +(1-\sqrt{\tau}) \mathbb{D} e\left(\frac{y_{\tau}^{k}-y_{\tau}^{k-1}}{\tau}\right): e(y)+\tau^{3 / 2} \mathbb{D} e\left(\frac{y-y_{\tau}^{k-1}}{\tau}\right): e\left(\frac{y-y_{\tau}^{k-1}}{\tau}\right) \\
& +\mu(1-\sqrt{\tau}) \nabla^{2} \frac{y_{\tau}^{k}-y_{\tau}^{k-1}}{\tau}: \nabla^{2} y+\mu \tau^{3 / 2} \nabla^{2} \frac{y-y_{\tau}^{k-1}}{\tau}: \nabla^{2} \frac{y-y_{\tau}^{k-1}}{\tau} \\
& +\varphi_{0}(\nabla y, \lambda)+\frac{\nu}{2}\left|\nabla^{2} y\right|^{2}+\frac{\beta}{2}|\nabla \lambda|^{2}+\mathscr{T}\left(w_{\tau}^{k}\right) \varphi_{1}^{\prime}\left(\nabla y_{\tau}^{k}\right): \nabla y \mathrm{~d} x \\
\text { subject to } & (y, \lambda) \in W^{1, \gamma}\left(\Omega ; \mathbb{R}^{3}\right) \times V,\left.\quad y\right|_{\Gamma_{0}}=y_{\mathrm{D}} .
\end{aligned}
$$

Due to the assumed mode of a certain "partial semi-convexity" (4.2c) implying the mentioned strict convexity if $\tau$ is small as specified, (4.7) has a convex coercive functional and possesses therefore a solution, let us denote it as $\left(\tilde{y}_{\tau}^{k}, \tilde{\lambda}_{\tau}^{k}\right)$, which is determined uniquely if $\left(y_{\tau}^{k}, w_{\tau}^{k}\right)$ is fixed.

Comparing optimality conditions for $(4.7)$ with $(4.3 \mathrm{a}, \mathrm{b})$ and taking into account the strict monotonicity of the underlying elliptic operator, we get $\tilde{y}_{\tau}^{k}=y_{\tau}^{k}$ and $\tilde{\lambda}_{\tau}^{k}=\lambda_{\tau}^{k}$. Then we can test (4.7) by $\left(y_{\tau}^{k-1}, \lambda_{\tau}^{k-1}\right)$, which yields the following discrete mechanical energy balance (as an inequality) holds:

$$
\begin{aligned}
\int_{\Omega} \frac{\varrho}{2} \mid & \left.\frac{y_{\tau}^{k}-y_{\tau}^{k-1}}{\tau}\right|^{2}+\varphi_{0}\left(\nabla y_{\tau}^{k}, \lambda_{\tau}^{k}\right)+\frac{\beta}{2}\left|\nabla \lambda_{\tau}^{k}\right|^{2}+\frac{\nu}{2}\left|\nabla^{2} y_{\tau}^{k}\right|^{2} \\
& +\frac{\tau}{\gamma}\left|\nabla^{2} y_{\tau}^{k}\right|^{\gamma}+\tau \sum_{l=1}^{k}\left(\zeta\left(\frac{\lambda_{\tau}^{l}-\lambda_{\tau}^{l-1}}{\tau}\right)\right. \\
& \left.+(1-\sqrt{\tau}) \mathbb{D} e\left(\frac{y_{\tau}^{l}-y_{\tau}^{l-1}}{\tau}\right): e\left(\frac{y_{\tau}^{l}-y_{\tau}^{l-1}}{\tau}\right)+\mu\left|\nabla^{2} \frac{y_{\tau}^{l}-y_{\tau}^{l-1}}{\tau}\right|^{2}\right) \mathrm{d} x \\
\leq & \int_{\Omega} \frac{\varrho}{2}\left|\dot{y}_{0}\right|^{2}+\varphi_{0}\left(\nabla y_{0}, \lambda_{0}\right)+\frac{\beta}{2}\left|\nabla \lambda_{0}\right|^{2}+\frac{\nu}{2}\left|\nabla^{2} y_{0}\right|^{2}+\frac{\tau}{\gamma}\left|\nabla^{2} y_{0}\right|^{\gamma} \\
& +\tau \sum_{l=1}^{k}\left(f_{\tau}^{l} \cdot \frac{y_{\tau}^{l}-y_{\tau}^{l-1}}{\tau}+\mathscr{T}\left(w_{\tau}^{l}\right) \varphi_{1}^{\prime}\left(\nabla y_{\tau}^{l}\right): \nabla \frac{y_{\tau}^{l}-y_{\tau}^{l-1}}{\tau}\right) \mathrm{d} x
\end{aligned}
$$

as well as the following discrete total energy balance

$$
\begin{aligned}
\int_{\Omega} & \frac{\varrho}{2}\left|\frac{y_{\tau}^{k}-y_{\tau}^{k-1}}{\tau}\right|^{2}+\varphi_{0}\left(\nabla y_{\tau}^{k}, \lambda_{\tau}^{k}\right)+\frac{\nu}{2}\left|\nabla^{2} y_{\tau}^{k}\right|^{2}+\frac{\beta}{2}\left|\nabla \lambda_{\tau}^{k}\right|^{2}+w_{\tau}^{k}+\frac{\tau}{\gamma}\left|\nabla^{2} y_{\tau}^{k}\right|^{\gamma} \mathrm{d} x \\
& \leq \int_{\Omega} \frac{\varrho}{2}\left|\dot{y}_{0}\right|^{2}+\varphi_{0}\left(\nabla y_{0}, \lambda_{0}\right)+\frac{\nu}{2}\left|\nabla^{2} y_{0}\right|^{2}+\frac{\beta}{2}\left|\nabla \lambda_{0}\right|^{2}+\frac{\tau}{\gamma}\left|\nabla^{2} y_{0}\right|^{\gamma}+w_{0} \\
& +\tau \sum_{l=1}^{k}\left(f_{\tau}^{l} \cdot \frac{y_{\tau}^{l}-y_{\tau}^{l-1}}{\tau}+\mathscr{S}\left(w_{\tau}^{l}, \nabla y_{\tau}^{l}, \lambda_{\tau}^{l}\right) \nabla \phi_{\tau}^{l} \cdot \nabla \phi_{\tau}^{l}\right) \mathrm{d} x+\tau \sum_{l=1}^{k} \int_{\Gamma} h_{\mathrm{ext}, \tau}^{l} \mathrm{~d} S
\end{aligned}
$$

and also the discrete "semistability"

$$
\int_{\Omega} \varphi_{0}\left(\nabla y_{\tau}^{k}, \lambda_{\tau}^{k}\right)+\frac{\beta}{2}\left|\nabla \lambda_{\tau}^{k}\right|^{2} \mathrm{~d} x \leq \int_{\Omega} \varphi_{0}\left(\nabla y_{\tau}^{k}, v\right)+\frac{\beta}{2}|\nabla v|^{2}+\xi_{1}\left(v-\lambda_{\tau}^{k}\right) \mathrm{d} x
$$

for any $v \in V$, and also the discrete electric-current continuity equation for $\phi_{\tau}^{k} \in$ $W^{1,2}(\Omega)$ satisfying the boundary conditions $\left.\phi_{\tau}^{k}\right|_{\Gamma_{0}}=\phi_{\mathrm{ext}, \tau}^{k}$ on $\Gamma_{0}$, namely

$$
\int_{\Omega} \mathscr{S}\left(w_{\tau}^{k}, \nabla y_{\tau}^{k}, \lambda_{\tau}^{k}\right) \nabla \phi_{\tau}^{k} \cdot \nabla v \mathrm{~d} x=0
$$

for any $k=1, \ldots, K_{\tau}$ and $v \in W_{\Gamma_{0}}^{1,2}(\Omega)$. Let us define the piecewise affine interpolant $\left(y_{\tau}, \lambda_{\tau}, w_{\tau}\right)$ by

$$
y_{\tau}(t):=\frac{t-(k-1) \tau}{\tau} y_{\tau}^{k}+\frac{k \tau-t}{\tau} y_{\tau}^{k-1} \quad \text { for } t \in[(k-1) \tau, k \tau],
$$


and similarly $\lambda_{\tau}(t)=\frac{t-(k-1) \tau}{\tau} \lambda_{\tau}^{k}+\frac{k \tau-t}{\tau} \lambda_{\tau}^{k-1}$ and $w_{\tau}(t)=\frac{t-(k-1) \tau}{\tau} w_{\tau}^{k}+\frac{k \tau-t}{\tau} w_{\tau}^{k-1}$ for $t \in[(k-1) \tau, k \tau]$ with $\stackrel{\tau}{k}=0, \ldots, K_{\tau}^{\tau}:=T / \tau$, assuming $K_{\tau} \stackrel{\tau}{\in} \mathbb{N}$. Also, we define the piecewise constant interpolant $\left(\bar{y}_{\tau}, \bar{\lambda}_{\tau}, \bar{w}_{\tau}\right)$ by

$$
\bar{y}_{\tau}(t):=y_{\tau}^{k}, \quad \bar{\lambda}_{\tau}(t):=\lambda_{\tau}^{k}, \quad \bar{w}_{\tau}(t):=w_{\tau}^{k} \quad \text { for } t \in((k-1) \tau, k \tau],
$$

for $k=0, \ldots, K_{\tau}$. Eventually, we define $\bar{f}_{\tau}$ and $\bar{\theta}_{\text {ext }, \tau}$ by $\left.\bar{f}_{\tau}\right|_{((k-1) \tau, k \tau]}:=f_{\tau}^{k}$ and recall that we already have defined $\left.\bar{\theta}_{\mathrm{ext}, \tau}\right|_{((k-1) \tau, k \tau]}:=\theta_{\mathrm{ext}, \tau}^{k}$. Occasionally, we will use also a "retarded" piecewise constant interpolant $\underline{w}_{\tau}$ defined by

$$
\underline{w}_{\tau}(t):=w_{\tau}^{k-1} \quad \text { for } t \in[(k-1) \tau, k \tau) .
$$

As to the approximated loading, we assume

$$
\begin{array}{ll}
\bar{f}_{\tau} \in L^{\infty}\left(I ; L^{2}\left(\Omega ; \mathbb{R}^{3}\right)\right), & \lim _{\tau \downarrow 0} \bar{f}_{\tau}=f \text { in } L^{1}\left(I ; L^{2}\left(\Omega ; \mathbb{R}^{3}\right)\right), \\
& \left\|\bar{f}_{\tau}\right\|_{L^{\infty}\left(I ; L^{2}\left(\Omega ; \mathbb{R}^{3}\right)\right)} \leq \frac{K}{\sqrt{\tau}}, \\
\bar{h}_{\mathrm{ext}, \tau} \in L^{\infty}(\Sigma), & \lim _{\tau \downarrow 0} \bar{h}_{\mathrm{ext}, \tau}=h_{\mathrm{ext}} \text { in } L^{1}(\Sigma), \quad \bar{h}_{\mathrm{ext}, \tau} \geq 0, \\
\bar{\phi}_{\mathrm{ext}, \tau} \in L^{\infty}\left(I ; W^{1 / 2,2}\left(\Gamma_{0}\right)\right), & \lim _{\tau \downarrow 0} \bar{\phi}_{\mathrm{ext}, \tau}=\phi_{\mathrm{ext}} \text { in } L^{2}\left(I ; W^{1 / 2,2}\left(\Gamma_{0}\right)\right) .
\end{array}
$$

The next step is to obtain the following a-priori estimates:

$$
\begin{aligned}
& \left\|y_{\tau}\right\|_{W^{1, \infty}\left(I ; L^{2}\left(\Omega ; \mathbb{R}^{3}\right)\right) \cap W^{1,2}\left(I ; W_{\Gamma_{0}}^{2,2}\left(\Omega ; \mathbb{R}^{3}\right)\right)} \leq C, \\
& \left\|\bar{\lambda}_{\tau}\right\|_{L^{\infty}(I ; V) \cap \operatorname{BV}\left(\bar{I} ; L^{1}\left(\Omega ; \mathbb{R}^{L}\right)\right)} \leq C, \\
& \left\|w_{\tau}\right\|_{L^{\infty}\left(I ; L^{1}(\Omega)\right) \cap L^{r}\left(I ; W^{1, r}(\Omega)\right)} \leq C \text { with any } 1 \leq r<5 / 4 \\
& \left\|\bar{\phi}_{\tau}\right\|_{L^{2}\left(I ; W^{1,2}(\Omega)\right)} \leq C \\
& \left\|\dot{w}_{\tau}\right\|_{L^{1}\left(I ; W^{3,2}(\Omega)^{*}\right)} \leq C, \\
& \left\|\dot{y}_{\tau}\right\|_{\mathrm{BV}\left(\bar{I} ; W_{\Gamma_{0}}^{2, \infty}\left(\Omega ; \mathbb{R}^{3}\right)^{*}\right)} \leq C \\
& \left\|y_{\tau}\right\|_{L^{\infty}\left(I ; W^{2, \gamma}\left(\Omega ; \mathbb{R}^{3}\right)\right)} \leq \frac{C}{\sqrt[\gamma]{\tau}}
\end{aligned}
$$

note that $\frac{\partial^{2}}{\partial t^{2}} y_{\tau}$ is a measure because $\frac{\partial}{\partial t} y_{\tau}$ is piecewise constant hence (4.16f) indeed cannot be improved by replacing "BV" by $L^{2}$, as possible for the limit "continuous" solution.

The estimate (4.16d) follows trivially under our data qualification by testing (4.11) by $\phi_{\tau}^{k}-\tilde{\phi}_{\mathrm{ext}, \tau}^{k}$ where $\tilde{\phi}_{\mathrm{ext}, \tau}^{k} \in W^{1,2}(\Omega)$ is a prolongation of $\phi_{\mathrm{ext}, \tau}^{k}$. The first estimates in (4.16a,b,c) and the estimate (4.16g) can be obtained from (4.9) by using a discrete Gronwall inequality. As $\nu>0$, we get now also $\bar{y}_{\tau}$ estimated in $L^{\infty}\left(I ; W^{2,2}\left(\Omega ; \mathbb{R}^{3}\right)\right)$.

Estimation of $\nabla \bar{w}_{\tau}$ in $L^{r}\left(Q ; \mathbb{R}^{3}\right)$ in (4.16c) is the highly delicate and it combines the technique proposed by Boccardo and Gallouët $[7,8]$ yielding

$$
\left\|\nabla \bar{w}_{\tau}\right\|_{L^{r}\left(Q ; \mathbb{R}^{3}\right)}^{r} \leq C\left(1+\left\|\bar{r}_{\tau}\right\|_{L^{1}(Q)}\right), \quad 1 \leq r<5 / 4,
$$

with a fine interpolation of the adiabatic term by using the already obtained $L^{\infty}(I ; \cdot)$-estimates; in (4.17), we have denoted by $\bar{r}_{\tau}$ the piece-wise constant interpolation of the right-hand side of the enthalpy equation (4.3c). It is important that we already have proved the Joule heat $\mathscr{S}\left(\bar{w}_{\tau}, \nabla \bar{y}_{\tau}, \bar{\lambda}_{\tau}\right) \nabla \bar{\phi}_{\tau} \cdot \nabla \bar{\phi}_{\tau}$ a-priori bounded in $L^{1}(Q)$. Adding (4.17) with a sufficiently small weight to the mechanical energy 
balance (4.8) summed for $k=1, \ldots, K_{\tau}$, we obtain estimate of the type

$$
\begin{aligned}
& \left\|\nabla^{2} \dot{y}_{\tau}\right\|_{L^{2}\left(Q ; \mathbb{R}^{3 \times 3 \times 3}\right)}^{2}+\left\|\dot{\lambda}_{\tau}\right\|_{L^{1}\left(Q ; \mathbb{R}^{L}\right)}+\left\|\nabla \bar{w}_{\tau}\right\|_{L^{r}\left(Q ; \mathbb{R}^{3}\right)}^{r} \\
& \leq C\left(1+\left|\int_{Q} \mathscr{T}\left(\bar{w}_{\tau}\right) \varphi_{1}^{\prime}\left(\nabla \bar{y}_{\tau}\right): \nabla \dot{y}_{\tau} \mathrm{d} x \mathrm{~d} t\right|\right)=: C(1+A) .
\end{aligned}
$$

Now, using at most linear growth of $\mathscr{T}$ and $(4.2 \mathrm{j})$, by Hölder's and Young's inequalities, we estimate

$$
\begin{aligned}
A & \leq \frac{1}{\inf c_{\mathrm{V}}(\cdot)} \int_{0}^{T}\left\|\bar{w}_{\tau}(t)\right\|_{L^{6 / 5}(\Omega)}\left\|\varphi_{1}^{\prime}\left(\nabla \bar{y}_{\tau}(t)\right)\right\|_{L^{\infty}\left(\Omega ; \mathbb{R}^{3 \times 3}\right)}\left\|\nabla \dot{y}_{\tau}(t)\right\|_{L^{6}\left(\Omega ; \mathbb{R}^{3 \times 3}\right)} \mathrm{d} t \\
& \leq \frac{\sup \left|\varphi_{1}^{\prime}\left(\mathbb{R}^{3 \times 3}\right)\right|}{2 \inf c_{\mathrm{V}}(\cdot)}\left(\frac{\left\|\bar{w}_{\tau}\right\|_{L^{2}\left(I ; L^{6 / 5}(\Omega)\right)}^{2}}{\delta_{1}}+\delta_{1}\left\|\nabla \dot{y}_{\tau}\right\|_{L^{2}\left(I ; L^{6}\left(\Omega ; \mathbb{R}^{3 \times 3}\right)\right)}^{2}\right)
\end{aligned}
$$

where we used (4.2j). After using the constant-in-time Dirichlet boundary conditions for $y_{\tau}$ (hence for $\frac{\partial}{\partial t} y_{\tau}$ too), we can estimate the last term in (4.19) by $\left\|\nabla^{2} \dot{y}_{\tau}\right\|_{L^{2}\left(Q ; \mathbb{R}^{3 \times 3 \times 3}\right)}^{2}$ and then absorb it in the first term in (4.18) if $\delta_{1}>0$ is chosen small enough.

Further, we interpolate $L^{6 / 5}(\Omega)$ between $L^{1}(\Omega)$ and $W^{1, r}(\Omega)$ by the GagliardoNirenberg inequality, $\left\|\bar{w}_{\tau}(t)\right\|_{L^{6 / 5}(\Omega)} \leq C\left(1+\left\|\nabla \bar{w}_{\tau}(t)\right\|_{L^{r}\left(\Omega ; \mathbb{R}^{3}\right)}\right)^{r_{1}}$ with $\frac{5}{6}>1-\frac{8}{15} r_{1}$; here again $r<5 / 4$ has been used. Thus we can choose $5 / 16<r_{1}<5 / 8$. Rising it to 2 , using $2 r_{1}<r$, and integrating over $I$, we obtain

$$
\left\|\bar{w}_{\tau}\right\|_{L^{2}\left(I ; L^{q}(\Omega)\right)}^{2} \leq \int_{0}^{T} C\left(1+\left\|\nabla \bar{w}_{\tau}(t)\right\|_{L^{r}\left(\Omega ; \mathbb{R}^{3}\right)}\right)^{2 r_{1}} \mathrm{~d} t \leq C_{\delta_{2}}+\delta_{2}\left\|\nabla \bar{w}_{\tau}(t)\right\|_{L^{r}\left(Q ; \mathbb{R}^{3}\right)}^{r}
$$

Having $\delta_{1}$ already fixed and taking $\delta_{2}>0$ sufficiently small, we can absorb $\delta_{2}\left\|\nabla \bar{w}_{\tau}(t)\right\|_{L^{r}\left(Q ; \mathbb{R}^{3}\right)}^{r}$ in the left-hand side of (4.18).

Thus the estimates (4.16a-c) are completed, and also the estimates (4.16d,g) then follow standardly from them.

The final and still pretty nontrivial step is the proof of convergence to some sort of a weak solution. Here, it means a limit passage in the (weakly formulated) discrete mechanical equilibrium (as an analog of (3.13a))

$$
\begin{aligned}
& \left.\left.\int_{Q}\left(\left[\varphi_{0}\right]_{F}^{\prime}\left(\nabla \bar{y}_{\tau}, \bar{\lambda}_{\tau}\right)+\mathscr{T}\left(\bar{w}_{\tau}\right) \varphi_{1}^{\prime}\left(\nabla \bar{y}_{\tau}\right)\right)\right): \nabla \bar{v}_{\tau}+\mathbb{D} e\left(\dot{y}_{\tau}\right)\right): e\left(\bar{v}_{\tau}\right) \\
& +\left(\left(\nu+\tau\left|\nabla^{2} \bar{y}_{\tau}\right|^{\gamma-2}\right) \nabla^{2} \bar{y}_{\tau}+\mu \nabla^{2} \dot{y}_{\tau}\right) \vdots \nabla^{2} \bar{v}_{\tau}-\bar{f}_{\tau} \cdot \bar{v}_{\tau} \mathrm{d} x \mathrm{~d} t \\
& \quad-\int_{\tau}^{T} \int_{\Omega} \varrho \dot{y}_{\tau}(\cdot-\tau) \cdot \frac{\partial v_{\tau}}{\partial t} \mathrm{~d} x \mathrm{~d} t+\int_{\Omega} \varrho \dot{y}_{\tau}(T) \cdot v_{\tau}(T) \mathrm{d} x=\int_{\Omega} \varrho \dot{y}_{0} \cdot v_{\tau}(\tau) \mathrm{d} x,
\end{aligned}
$$

where $\bar{v}_{\tau}$ and $v_{\tau}$ denote respectively the piecewise constant and the piecewise affine interpolants of the $\left\{v^{k}\right\}_{k=0}^{K_{\tau}}$ on the equidistant partition of $[0, T]$ with the time step $\tau$. Further, we need a limit passage in the (weakly formulated) discrete enthalpy equation

$$
\begin{aligned}
\int_{\Omega} w_{\tau}(T) v(T) \mathrm{d} x+\int_{Q} \mathscr{K}\left(\nabla \bar{y}_{\tau}, \bar{\lambda}_{\tau}, \bar{w}_{\tau}\right) \nabla \bar{w}_{\tau} \cdot \nabla v \\
-\left(\zeta\left(\frac{\partial \lambda_{\tau}}{\partial t}\right)+(1-\sqrt{\tau}) \mathbb{D} e\left(\dot{y}_{\tau}\right): e\left(\dot{y}_{\tau}\right)+\mu\left|\nabla^{2} \dot{y}_{\tau}\right|^{2}\right. \\
\left.+\mathscr{S}\left(\bar{w}_{\tau}, \nabla \bar{y}_{\tau}, \bar{\lambda}_{\tau}\right) \nabla \bar{\phi}_{\tau} \cdot \nabla \bar{\phi}_{\tau}+\mathscr{T}\left(\bar{w}_{\tau}\right) \varphi_{1}^{\prime}\left(\nabla \bar{y}_{\tau}\right): \nabla \dot{y}_{\tau}\right) v \mathrm{~d} x \mathrm{~d} t \\
-\int_{\tau}^{T} \int_{\Omega} \underline{w}_{\tau} \frac{\partial v}{\partial t} \mathrm{~d} x \mathrm{~d} t=\int_{\Sigma} \bar{h}_{\mathrm{ext}, \tau} v \mathrm{~d} S \mathrm{~d} t+\int_{\Omega} w_{0} v(\tau, \cdot) \mathrm{d} x
\end{aligned}
$$


further in the discrete energy balance (as an inequality)

$$
\begin{aligned}
\int_{\Omega} & \frac{\varrho}{2}\left|\dot{y}_{\tau}(T)\right|^{2}+\varphi_{0}\left(\nabla y_{\tau}(T), \lambda_{\tau}(T)\right)+\frac{\beta}{2}\left|\nabla \bar{\lambda}_{\tau}(T)\right|^{2}+\frac{\nu}{2}\left|\nabla^{2} y_{\tau}(T)\right|^{2}+w_{\tau}(T) \mathrm{d} x \\
& \leq \int_{Q} \bar{f}_{\tau} \cdot \dot{y}_{\tau}+\mathscr{S}\left(\bar{w}_{\tau}, \nabla \bar{y}_{\tau}, \bar{\lambda}_{\tau}\right) \nabla \bar{\phi}_{\tau} \cdot \nabla \bar{\phi}_{\tau} \mathrm{d} x \mathrm{~d} t+\int_{\Omega} \frac{\varrho}{2}\left|\dot{y}_{0}\right|^{2}+\varphi_{0}\left(\nabla y_{0}, \lambda_{0}\right) \\
& +\frac{\beta}{2}\left|\nabla \bar{\lambda}_{0}\right|^{2}+\frac{\nu}{2}\left|\nabla^{2} y_{0}\right|^{2}+h_{0}\left(\theta_{0}\right)+\frac{\tau}{\gamma}\left|e\left(u_{0}\right)\right|^{\gamma} \mathrm{d} x+\int_{\Sigma} \bar{h}_{\text {ext }, \tau} \mathrm{d} S \mathrm{~d} t,
\end{aligned}
$$

the discrete semistability integrated over $I$ :

$$
\int_{Q} \varphi_{0}\left(\nabla \bar{y}_{\tau}, \bar{\lambda}_{\tau}\right)+\frac{\beta}{2}\left|\nabla \bar{\lambda}_{\tau}\right|^{2} \mathrm{~d} x \mathrm{~d} t \leq \int_{Q} \varphi_{0}\left(\nabla \bar{y}_{\tau}, v\right)+\frac{\beta}{2}|\nabla v|^{2}+\xi_{1}\left(v-\bar{\lambda}_{\tau}\right) \mathrm{d} x \mathrm{~d} t
$$

for any $v \in L^{\infty}(I ; V)$, and discrete electric-current continuity equation integrated over $I$ :

$$
\int_{Q} \mathscr{S}\left(\bar{w}_{\tau}, \nabla \bar{y}_{\tau}, \bar{\lambda}_{\tau}\right) \nabla \bar{\phi}_{\tau} \cdot \nabla v \mathrm{~d} x \mathrm{~d} t=0
$$

for any $v \in L^{2}\left(I ; W_{\Gamma_{0}}^{1,2}(\Omega)\right)$.

Then, by Banach selection principle, the a-priori estimates (4.16a-g) allow for selection of weakly* converging subsequence converging in the spaces indicated in $(4.16 \mathrm{a}-\mathrm{g})$ to a limit, let us denote it by $(y, \lambda, \theta, \phi)$.

Estimates of the gradients cause, by Aubin-Lions' compact-embedding theorem (possibly generalized for time-derivative as measures [47, Corollary 7.9]), strong convergence of $\nabla \bar{y}_{\tau}, \bar{w}_{\tau}$, and, in case $\beta>0$, also $\bar{\lambda}_{\tau}$. We also obtain strong convergence of $\bar{\phi}_{\tau} \rightarrow \phi$ in $W^{1,2}(\Omega)$ from the estimate

$$
\begin{aligned}
\alpha_{\mathbb{S}}\left\|\nabla\left(\bar{\phi}_{\tau}-\phi\right)\right\|_{L^{2}\left(\Omega ; \mathbb{R}^{3}\right)}^{2} & \leq \int_{\Omega} \mathscr{S}\left(\bar{w}_{\tau}, \nabla \bar{y}_{\tau}, \bar{\lambda}_{\tau}\right) \nabla\left(\bar{\phi}_{\tau}-\phi\right) \cdot \nabla\left(\bar{\phi}_{\tau}-\phi\right) \mathrm{d} x \\
& =\int_{\Omega} \mathscr{S}\left(\bar{w}_{\tau}, \nabla \bar{y}_{\tau}, \bar{\lambda}_{\tau}\right) \nabla \phi \cdot \nabla\left(\phi-\bar{\phi}_{\tau}\right) \mathrm{d} x \\
& +\int_{\Omega} \mathscr{S}\left(\bar{w}_{\tau}, \nabla \bar{y}_{\tau}, \bar{\lambda}_{\tau}\right) \nabla \bar{\phi}_{\tau} \cdot \nabla\left(\tilde{\phi}_{\text {ext }}-\tilde{\bar{\phi}}_{\text {ext }, \tau}\right) \mathrm{d} x \rightarrow 0
\end{aligned}
$$

with $\alpha_{\mathbb{S}}$ from (4.2l), where we used (4.24) tested by $v:=\bar{\phi}_{\tau}-\phi+\tilde{\phi}_{\text {ext }}-\tilde{\bar{\phi}}_{\text {ext }, \tau}$ where the tilde denotes a suitable extension on $Q$ satisfying $\tilde{\phi}_{\text {ext }}-\tilde{\bar{\phi}}_{\text {ext }, \tau} \rightarrow 0$ in $L^{2}\left(I ; W^{1,2}(\Omega)\right)$; note that $\left.v(t)\right|_{\Gamma_{0}}=0$ so that $v$ is a legal test function. Thus the limit passage in (4.24) is simple and, for (4.22) and (4.21), it is also important that the Joule heat $\mathscr{S}\left(\bar{w}_{\tau}, \nabla \bar{y}_{\tau}, \bar{\lambda}_{\tau}\right) \nabla \bar{\phi}_{\tau} \cdot \nabla \bar{\phi}_{\tau}$ converges to $\mathscr{S}(w, \nabla y, \lambda) \nabla \phi \cdot \nabla \phi$ even strongly in $L^{1}(Q)$.

Limit passage in the mechanical equilibrium (4.20) is then just by continuity. It is important that the regularizing term vanishes because, employing $(4.16 \mathrm{~g})$, we have

$$
\begin{gathered}
\left.\left|\int_{Q} \tau\right| \nabla^{2} \bar{u}_{\tau}\right|^{\gamma-2} \nabla^{2} \bar{u}_{\tau} \vdots \nabla^{2} v \mathrm{~d} x \mathrm{~d} t \mid \leq \tau\left\|\nabla^{2} \bar{u}_{\tau}\right\|_{L^{\gamma}\left(Q ; \mathbb{R}^{3 \times 3 \times 3}\right)}^{\gamma-1}\left\|\nabla^{2} v\right\|_{L^{\gamma}\left(Q ; \mathbb{R}^{3 \times 3 \times 3}\right)} \\
\leq \tau\left(\frac{C}{\sqrt[\gamma]{\tau}}\right)^{\gamma-1}\left\|\nabla^{2} v\right\|_{L^{\gamma}\left(Q ; \mathbb{R}^{3 \times 3 \times 3}\right)}=\mathscr{O}(\sqrt[\gamma]{\tau}) \rightarrow 0
\end{gathered}
$$

Also limit passage in the energy balance (4.22) and semi-stability (4.23) is then simply by (lower semi-) continuity. The last delicate point is limit passage in the enthalpy equation (4.21). Here it is important that we have already proved the limit semi-stability and, in addition, we can prove that $\ddot{y}$ is not only a $W_{\Gamma_{0}}^{2, \infty}\left(\Omega ; \mathbb{R}^{3}\right)^{*}$-valued measure as suggested by $(4.16 \mathrm{f})$, but even it belongs even to $L^{2}\left(I ; W_{\Gamma_{0}}^{2,2}\left(\Omega ; \mathbb{R}^{3}\right)^{*}\right)+L^{1}\left(I ; L^{2}\left(\Omega ; \mathbb{R}^{3}\right)\right)$ and is thus in duality with $\frac{\partial}{\partial t} y \in$ 
$L^{2}\left(I ; W_{\Gamma_{0}}^{2,2}\left(\Omega ; \mathbb{R}^{3}\right)\right) \cap L^{\infty}\left(I ; L^{2}\left(\Omega ; \mathbb{R}^{3}\right)\right)$, cf. (4.16a). Hence, using the advanced results from the theory of rate-independent processes $[12,15,27,28]$, we can prove the energy balance as an equality in the limit. This allows for showing that the overall integral dissipated energy must converge, from which we get strong convergence of the viscous contributions and weak* convergence of the rate-independent contributions, cf. [50, Formulas (4.69)-(4.75) with (4.83)] for these very technical details; here $(4.2 \mathrm{~h}, \mathrm{i}, \mathrm{n})$ is needed. Then the limit passage in $(4.21)$ is easy.

If $\beta=0$, we must rely on the weak* convergence of $\lambda_{\tau} \rightarrow \lambda$ in $L^{\infty}\left(I ; L^{2}\left(\Omega ; \mathbb{R}^{L}\right)\right)$. This is why we had to assume $\mathbb{S}$ and $\mathbb{K}$ independent of $\lambda$ in (4.2q), so that $\mathscr{S}$ and $\mathscr{K}$ independent of $\lambda$, too. The only delicate point is then the limit passage in semi-stability (4.23) that can rely on the quadratic form of $\varphi_{0}$, cf. (4.2q) and the "binomial" formula $\varphi_{0}\left(F, \lambda_{1}\right)-\varphi_{0}\left(F, \lambda_{2}\right)=\frac{1}{2}\left[\varphi_{0}\right]_{\lambda}^{\prime}\left(F, \lambda_{1}-\lambda_{2}\right)\left(\lambda_{1}+\lambda_{2}\right)$, so that, for a test function $v_{\tau}=v-\lambda+\bar{\lambda}_{\tau}$ for (4.23), we get

$$
\begin{gathered}
\int_{Q} \varphi_{0}\left(\nabla \bar{y}_{\tau}, \bar{\lambda}_{\tau}\right) \mathrm{d} x \mathrm{~d} t-\int_{Q} \varphi_{0}\left(\nabla \bar{y}_{\tau}, v_{\tau}\right) \mathrm{d} x \mathrm{~d} t=\int_{Q}\left[\varphi_{0}\right]_{\lambda}^{\prime}\left(\nabla \bar{y}_{\tau}, \bar{\lambda}_{\tau}-v_{\tau}\right) \frac{\bar{\lambda}_{\tau}+v_{\tau}}{2} \mathrm{~d} x \mathrm{~d} t \\
=\int_{Q}\left[\varphi_{0}\right]_{\lambda}^{\prime}\left(\nabla \bar{y}_{\tau}, \lambda-v\right) \frac{\bar{\lambda}_{\tau}+v_{\tau}}{2} \mathrm{~d} x \mathrm{~d} t \rightarrow \int_{Q}\left[\varphi_{0}\right]_{\lambda}^{\prime}(\nabla y, \lambda-v) \frac{\lambda+v}{2} \mathrm{~d} x \mathrm{~d} t \\
=\int_{Q} \varphi_{0}(\nabla y, \lambda) \mathrm{d} x \mathrm{~d} t-\int_{Q} \varphi_{0}(\nabla y, v) \mathrm{d} x \mathrm{~d} t,
\end{gathered}
$$

where we used also the strong convergence $\nabla \bar{y}_{\tau} \rightarrow \nabla y$ in $L^{1 / \epsilon}\left(I ; L^{6-\epsilon}\left(\Omega ; \mathbb{R}^{3 \times 3}\right)\right)$ for any $\epsilon>0$. In the context of rate-independent processes, this "binomial trick" was pronounced in [31]. Also, it is important that $\left[\phi_{0}\right]_{F}^{\prime}(F, \cdot)$ in $(4.20)$ is affine due to the special ansatz (4.2q) so that the weak* convergence $\lambda_{\tau} \rightarrow \lambda$ works also for limiting (4.20).

Thus we proved existence of a so-called energetic solution $(y, \lambda, w, \phi)$ to the system (3.13) with the boundary conditions (3.17)-(3.18)-(3.19) and the initial conditions (3.14) which is defined just to satisfy initial conditions and the five identities like (4.20)-(4.24) but with the indices $\tau$ and with $\gamma$-terms omitted. Also the estimates like (4.16a-e) are inherited from the discrete approximate solution. More specifically, we proved:

Proposition 4.1. Let the data $\varphi_{0}, \varphi_{1}, c_{\mathrm{v}}, \mathscr{K}, \mathscr{S}, f, y_{\mathrm{D}}, h_{\mathrm{ext}}, \phi_{\mathrm{ext}}, y_{0}, \dot{y}_{0}, \lambda_{0}$, and $\theta_{0}$ satisfy the qualification (4.2). Then the system (3.13) with the initial/boundary conditions (3.14)-(3.17)-(3.18)-(3.19) admits an energetic solution $(y, \lambda, w, \phi)$ with

$$
\begin{aligned}
& y \in W^{1, \infty}\left(I ; L^{2}\left(\Omega ; \mathbb{R}^{3}\right)\right), \cap W^{1,2}\left(I ; W_{\Gamma_{0}}^{2,2}\left(\Omega ; \mathbb{R}^{3}\right)\right), \\
& \lambda \in L^{\infty}(I ; V) \cap \operatorname{BV}\left(\bar{I} ; L^{1}\left(\Omega ; \mathbb{R}^{L}\right)\right) \text { with } V \text { from }(4.1), \\
& w \in L^{\infty}\left(I ; L^{1}(\Omega)\right) \cap L^{r}\left(I ; W^{1, r}(\Omega)\right) \text { with } 1 \leq r<5 / 4, \\
& \phi \in L^{2}\left(I ; W^{1,2}(\Omega)\right) .
\end{aligned}
$$

Moreover,

$$
\begin{aligned}
& \dot{w} \in L^{1}\left(I ; W^{3,2}(\Omega)^{*}\right), \text { and } \\
& \ddot{y} \in L^{2}\left(I ; W_{\Gamma_{0}}^{2,2}\left(\Omega ; \mathbb{R}^{3}\right)^{*}\right)+L^{1}\left(I ; L^{2}\left(\Omega ; \mathbb{R}^{3}\right)\right) .
\end{aligned}
$$

Remark 4.2. (Justification of the energetic-solution concept.) It is important (though not obvious) that the above definition of a solution is indeed selective in the sense that if this energetic solution is, in addition, smooth, then it actually satisfies (3.13) with (3.14) and (3.17)-(3.18)-(3.19), cf. [50, Proposition 3.2] or, for the isothermal case, also [49, Proposition 5.2].

Remark 4.3. (Numerical implementation.) Let us just remark that, to use the recursive system (4.3) for computer calculations of a solution, one must use also a spatial 
discretization (e.g. by a finite-element method with P2-elements) and an iterative solver because (4.3) is unfortunately fully coupled system without any variational structure (contrary to an isothermal case). Modification by using a nonlocal regularizing term instead of $\nabla F$-term would allow for using simply conventional P1-finite elements, cf. [3, 4]. On the other hand, 3D numerical simulations in [3] reveals that application of such model to real experiments requires great effort for both code development, computations, and fitting with experiments and the applicability in this sense is quite limited mainly because of the multiscale multidimensional multifield character of the problem. A computational efficient method for fighting with the multiscale character implements the microstructure typically created during evolution by so-called Young measures, cf. [25, 26, 46], but its adaptation to the multifield model presented here has not been done yet.

Remark 4.4. (General load.) Time-varying Dirichlet boundary conditions $y_{\mathrm{D}}$ would correspond certainly to reality of "hard-device" load in physical experiments much better than the constant $y_{\mathrm{D}}$ we considered above. After a usual shift, one could transform the problem to the above situation but the explicit time dependence would thus emerge in the constitutive potentials $\phi$ and $\xi$ 's. All analytical considerations would become technically much more complicated. Standardly, $W^{1,1}$-smoothness in time has to be assumed, cf. [15]. This applies also to a nonvanishing load $g$ in (3.17), although it makes less complicated expansion than the Dirichlet load $y_{\mathrm{D}}$.

\section{References}

[1] T. Aiki: Weak solutions for Falk's model of shape memory alloys. Math. Meth. Appl. Sci. 23 (2000), 299-319.

[2] L. Anand and M.E. Gurtin: Thermal effects in the superelasticity of crystalline shapememory materials. J. Mech. Phys. Solids 51 (2003), 1015-1058.

[3] M. Arndt, M.Griebel, V. Novák, T. Roubíček, P.Šittner: Martensitic/austenitic transformation in NiMnGa: simulation and experimental approaches, Int. J. Plasticity 22 (2006), 1943-1961.

[4] M. Arndt, M.Griebel, T. Roubíček: Modelling and numerical simulation of martensitic transformation in shape memory alloys. Cont. Mech. Thermodyn. 15 (2003), 463-485.

[5] J.M. Ball, R.D. James: Proposed experimental tests of a theory of fine microstructure and the two-well problem. Phil. Trans. Royal Soc. London A 338 (1992), 389-450.

[6] K. Bhattacharya: Microstructure of martensite. Why it forms and how it gives rise to the shape-memory effect. Oxford Univ. Press, New York, 2003.

[7] L. Boccardo, A. Dall'aglio, T. Gallouët, L. Orsina: Nonlinear parabolic equations with measure data. J. of Funct. Anal. 147 (1997), 237-258.

[8] L. Boccardo, T. Gallouët, Non-linear elliptic and parabolic equations involving measure data. J. Funct. Anal. 87 (1989), 149-169.

[9] M. Brokate, J. Sprekels: Hystreresis and Phase Transitions. Springer, New York, 1996.

[10] Z. Chen, K.-H. Hoffmann: On a one-dimensional nonlinear thermovisoelastic model for structural phase transitions in shape memory alloys. J. Diff. Eq. 12 (1994), 325-350.

[11] B.D. Coleman, W. Noll: The thermodynamics of elastic materials with heat conduction and viscosity, Arch. Rational Mech. Anal. 13 (1963), 167-178

[12] G. DalMaso, G.A. Francfort, R. Toader: Quasistatic crack growth in nonlinear elasticity. Arch. Rat. Mech. Anal. 176 (2005), 165-225.

[13] F. Falk: Model free energy, mechanics and thermodynamics of shape memory alloys. Acta Metallurgica 28 (1980), 1773-1780.

[14] M. G. Faulkner, J. J. Amalraj, A. Bhattacharyya: Experimental determination of thermal and electrical properties of NiTi shape memory wires. Smart Mater. Struct. 9 (2000), 632-639.

[15] G. Francfort and A. Mielke: An existence result for a rate-independent material model in the case of nonconvex energies. J. reine u. angew. Math. 595 (2006), 55-91. 
[16] M. Frémond: Non-Smooth Thermomechanics. Springer, Berlin, 2002.

[17] E. Fried and M. E. Gurtin: Dynamic solid-solid transitions with phase characterized by an order parameter Physica D: Nonlinear Phenomena 72 (1994), 287-308.

[18] H. Garcke: Travelling wave solutions as dynamic phase transitions in shape memory alloys. J. Diff. Eq. 121 (1995), 203-231.

[19] F. Gori, D. Carnevale, A. Doro Altan, S. Nicosia, E. Pennestrì: A new hysteretic behavior in the electrical resistivity of flexinol shape memory alloys versus temperature. Intl. J. Thermophysics 27 (2006), 866-879.

[20] B. Halphen, Q.S. Nguyen: Sur les matériaux standards généralisés. J. Mécanique 14 (1975), 39-63.

[21] Z. He, K.R. Gall, L.C. Brinson: Use of electric reistance testing to redefine the transformation kinetics and phase diagram for shape-mmory alloys. Metallurgical and materials transactions A 37A (2006), 579-581.

[22] K.-H. Hoffmann, A. Zochowski: Existence of solutions to some non-linear thermoelastic systems with viscosity. Math. Methods Appl. Sci. 15 (1992), 187-204.

[23] K. Hormann, J. Zimmer: On Landau theory and symmetric landscape for phase transitions. J. Mech. Physics Solids 55 (2007), 1385-1409.

[24] R.D. James, K.F. Hane: Martensitic transformations and shape-memory materials. Acta Mater. 48 (2000), 197-222.

[25] M. Kružík: Numerical approach to double-well problem. SIAM J. Numer. Analysis 35 (1998), 1833-1849.

[26] M. Kružík, A. Mielke, T. Roubíček: Modelling of microstructure and its evolution in shape-memory-alloy single-crystals, in particular in CuAlNi. Meccanica 40 (2005), $389-418$.

[27] A. Mielke: Evolution of rate-independent systems. In: Handbook of Differential Equations: Evolutionary Diff. Eqs. (Eds. C.Dafermos, E.Feireisl), Elsevier, Amsterdam, 2005, pp. 461-559.

[28] A. Mielke: A mathematical framework for generalized standard materials in rateindependent case. In: Multifield Problems in Fluid and Solid Mech. (Eds:R.Helmig et al.), Springer, 2006, 491-529.

[29] A. Mielke, F. Theil: On rate-independent hysteresis models. Nonlin. Diff. Eq. Appl. 11 (2004), 151-189.

[30] A. Mielke, F. Theil, V.I. Levitas: A variational formulation of rate-independent phase transformations using an extremum principle. Archive Rat. Mech. Anal. 162 (2002), $137-177$

[31] A. Mielke, A. Timofte: Two-scale homogenization for evolutionar variational inequalities via energetic formulation. SIAM J. Math. Anal. 39 (2007), 642-668.

[32] M. Niezgódka, J. Sprekels: Existence of solutions for a mathematical model of structural phase transitions in shape memory alloys. Math. Meth. Appl. Sci. 10 (1988), 197-223.

[33] V. Novák, P.Šittner, G.N. Dayananda, F.M. Braz-Fernandes, K.K. Mahesh: Electric resistance variation of $\mathrm{NiTi}$ shape memory alloy wires in thermomechanical tests: experiments and simulation. Mater. Sci. Engr. A 481-482 (2008), 127-133.

[34] L. Onsager: Reciprocal relations in irreversible processes. Phys. Rev. II 37 (1931), 405-426 and 38 (1931), 2265-2279.

[35] H.C.Öttinger: Beyond Equilibrium Thermodynamics. Wiley, Hoboken, NJ, 2002.

[36] I. Pawłow: Three-dimensional model of thermomechanical evolution of shape memory material. Control and Cybernetics 29 (2000), 341-365.

[37] I. Pawłow, W.M. Zajączkowski: Global existence to a three-dimensional non-linear thermoelasticity system arising in shape memory materials. Math. Methods Appl. Sci. 28 (2005), 407-442.

[38] I. Pawłow, A. Zochowski: Existence and uniqueness of solutions for a three-dimensional thermoelastic system. Dissertationes Math. (Rozprawy Mat.) 406 (2002), 46 pp.

[39] P. Plecháč, T. Roubíček: Visco-elasto-plastic model for martensitic phase transformation in shape-memory alloys. Math. Methods Appl. Sci. 25 (2002), 1281-1298. 
[40] M. Pitteri, G. Zanzotto: Continuum Models for Phase Transitions and Twinning in Crystals. Chapman \& Hall, Boca Raton, 2003.

[41] P. Podio-Guidugli: Contact interactions, stress, and material symmetry, for nonsimple elastic materials Theor. Appl. Mech. 28-29 (2002), 261-276.

[42] P. Podio-Guidugli: Models of phase segregation and diffusion of atomic species on a lattice Ric. Mat. 55 (2006), 105-118.

[43] K.R. Rajagopal, T. Roubíček: On the effect of dissipation in shape-memory alloys. Nonlinear Anal., Real World Appl. 4 (2003), 581-597.

[44] R. T. Rockafellar: Convex Analysis. Princeton University Press, Princeton, N.J., 1970.

[45] T. Roubíček: Dissipative evolution of microstructure in shape memory alloys. In: Lectures on Applied Mathematics. (H.-J. Bungartz, R. H. W. Hoppe, C. Zenger, eds.) Springer, Berlin, 2000, pp.45-63.

[46] T. Roubíček: Models of microstructure evolution in shape memory materials. In: Nonlin. Homogenization and Appl. to Composites, Polycrystals and Smart. Mater. (Eds. P.Ponte Castañeda, J.J.Telega, B.Gambin), NATO Sci.Ser. II/170, Kluwer, 2004, p.269-304.

[47] T. Roubíček: Nonlinear Partial Differential Equations with Applications. Birkhäuser, Basel, 2005.

[48] T. Roubíček: Modelling of thermodynamics of martensitic transformation in shapememory alloys. Disc. Cont. Dynam. Systems (2007), 892-902.

[49] T. Roubíček: Rate independent processes in viscous solids at small strains. Math. Meth. Appl. Sci. (2008), in print.

[50] T. Roubíček: Thermodynamics of rate independent processes in viscous solids at small strains. SIAM J. Math. Anal, submitted.

[51] J. Sprekels: Global existence for thermomechanical processes with nonconvex free energies of Ginzburg-Landau form. J. Math. Anal. Appl. 141, 333-348 (1989)

[52] J. Sprekels, S. Zheng: Global solutions to the equations of a Ginzburg-Landau theory for structural phase transitions in shape memory alloys. Physica D 39 (1989), 39-54.

[53] C. Trimarco: The structure of material forces in electromagnetic materials. Rend. Sem. Mat. Univ. Politec. Torino 58 (2000), 237-244.

[54] J. Uchil, K.K. Mahesh, K. Ganesh Kumara: Electrical resistivity and strain recovery studies on the effect of thermal cycling under constant strass on R-phase in NiTi shape memory alloy. Physica B 324 (2002), 419-428.

[55] X.D. Wu, Y.Z. Fan, J.S. Wu: A study on the variations of the eletrical resistance for NiTi shape memory alloy wires during the thermomechanical loading. Materials and Design 21 (2000), 511-515.

[56] J. Zimmer: Global existence of a nonlinear system in thermoviscoelasticity with nonconvex energy. J. Math. Anal. Appl. 292 (2004), 589-604.

\section{Acknowledgment}

The authors are thankful to Drs. Michal Landa and Petr Šittner for many inspiring discussions.

Tomáš Roubíček

Mathematical Institute, Charles University

Sokolovská 83, CZ-186 75 Praha 8, Czech Republic,

and

Institute of Thermomechanics of the ASCR,

Dolejškova 5, CZ-182 00 Praha 8, Czech Republic

e-mail: tomas.roubicek@mff.cuni.cz

Giuseppe Tomassetti

Dipartimento di Ingegneria Civile, Università di Roma "Tor Vergata"

Via di Tor Vergata 110, I-00133 Roma, Italy

e-mail: tomassetti@ing. uniroma2.it 